\title{
Synergy of radiotherapy and PD-1 blockade in Kras-mutant lung cancer
}

\author{
Grit S. Herter-Sprie, ${ }^{1,2,3}$ Shohei Koyama, ${ }^{1,2,4}$ Houari Korideck, ${ }^{5,6}$ Josephine Hai, ${ }^{1,2,3}$ Jiehui Deng, ${ }^{1,2,3}$ \\ Yvonne Y. Li, ${ }^{1,2,3}$ Kevin A. Buczkowski, ${ }^{1,2,3}$ Aaron K. Grant, ${ }^{7}$ Soumya Ullas, ${ }^{8}$ Kevin Rhee, ${ }^{1,2,3}$ \\ Jillian D. Cavanaugh, ${ }^{1,2,3}$ Neermala Poudel Neupane, ${ }^{1,2,3}$ Camilla L. Christensen, ${ }^{1,2,3}$ Jan M. Herter, ${ }^{9}$ \\ C. Mike Makrigiorgos, ${ }^{5,6}$ F. Stephen Hodi, ${ }^{1,2}$ Gordon J. Freeman, ${ }^{1,2,4}$ Glenn Dranoff, ${ }^{1,2,4}$ \\ Peter S. Hammerman, ${ }^{1,2,3}$ Alec C. Kimmelman, ${ }^{6,10}$ and Kwok-Kin Wong ${ }^{1,2,4,6}$ \\ 'Department of Medicine, Harvard Medical School, Boston, Massachusetts, USA. Department of Medical Oncology, \\ Dana-Farber Cancer Institute, Boston, Massachusetts, USA. ${ }^{3}$ Lowe Center for Thoracic Oncology, ${ }^{4}$ Cancer Vaccine Center, \\ ${ }^{5}$ Division of Medical Physics and Biophysics, and 6 Department of Radiation Oncology, Dana-Farber Cancer Institute, \\ Brigham and Women's Hospital, Harvard Medical School, Boston, Massachusetts, USA. 'Division of MRI Research, \\ Department of Radiology, and ${ }^{8}$ Longwood Small Animal Imaging Facility, Beth Israel Deaconess Medical Center, Harvard \\ Medical School, Boston, MA, USA. ${ }^{9}$ Center for Excellence in Vascular Biology, Department of Pathology, Brigham and \\ Women's Hospital, Harvard Medical School, Boston, MA, USA. ${ }^{10}$ Division of Genomic Stability and DNA Repair, \\ Dana-Farber Cancer Institute, Brigham and Women's Hospital, Harvard Medical School, Boston, Massachusetts, USA. \\ ${ }^{1 B} B$ elfer Institute for Applied Cancer Science, Dana-Farber Cancer Institute, Boston, Massachusetts, USA.
}

Authorship note: G.S. Herter-Sprie and S. Koyama contributed equally to this work.

Conflict of interest: G. Dranoff received sponsored research support from Novartis and Bristol-Myers Squibb. He currently is an employee of Novartis. F.S. Hodi has served as a consultant to Merck and Novartis and has received research support from Bristol-Myers Squibb. G.J. Freeman receives patent royalties (patent 6808710) on the PD-1 pathway from Bristol-Myers Squibb, Roche, Merck, EMD-Serrono, Boehringer Ingelheim, Amplimmune/ AstraZeneca, and Novartis.

Submitted: March 3, 2016 Accepted: May 17, 2016 Published: June 16, 2016

Reference information: JCI Insight. 2016;1(9):e87415. doi:10.1172/jci.insight.87415
Radiation therapy (RT), a critical modality in the treatment of lung cancer, induces direct tumor cell death and augments tumor-specific immunity. However, despite initial tumor control, most patients suffer from locoregional relapse and/or metastatic disease following RT. The use of immunotherapy in non-small-cell lung cancer (NSCLC) could potentially change this outcome by enhancing the effects of RT. Here, we report significant (up to $70 \%$ volume reduction of the target lesion) and durable (up to 12 weeks) tumor regressions in conditional Kras-driven genetically engineered mouse models (GEMMs) of NSCLC treated with radiotherapy and a programmed cell death 1 antibody ( $\alpha$ PD-1). However, while $\alpha$ PD-1 therapy was beneficial when combined with RT in radiation-naive tumors, $\alpha \mathrm{PD}-1$ therapy had no antineoplastic efficacy in RT-relapsed tumors and further induced $\mathrm{T}$ cell inhibitory markers in this setting. Furthermore, there was differential efficacy of $\alpha P D-1$ plus RT among Kras-driven GEMMs, with additional loss of the tumor suppressor serine/threonine kinase 11/liver kinase B1 (Stk11/Lkb1) resulting in no synergistic efficacy. Taken together, our data provide evidence for a close interaction among RT, T cells, and the PD-1/PD-L1 axis and underscore the rationale for clinical combinatorial therapy with immune modulators and radiotherapy.

\section{Introduction}

Despite recent advances in chemotherapy and molecular targeted therapy, lung cancer remains the leading cause of cancer-related deaths in the United States (1). Radiation therapy (RT) is a common nonsurgical treatment in the management of patients with non-small-cell lung carcinomas (NSCLC) that reduces disease recurrence and improves overall survival (2). The cytoreductive effectiveness of RT is a result of generating DNA damage, modulation of signal transduction, and promoting an inflamed tumor microenvironment attracting the host's immune system to the tumor site. However, the role of the inflammatory response still remains a double-edged sword. This inflammation can trigger innate and adaptive immune responses leading to tumor regression, but it can also enable tumor cells to develop multiple resistance mechanisms facilitating tumor immune escape (3). Recently, promising clinical antitumor effects have been reported with the incorporation of agents targeting the programmed cell death 1 (also known as CD279)/programmed death-ligand 1 (also known as CD274) (PD-1/PD-L1) immune checkpoint in NSCLC; however, there is a need to understand how to combine checkpoint blockade with other treatments, such as local therapy with RT $(4,5)$. Specifically, the recent work by Rizvi et al. showed increased therapeutic efficacy of anti-PD-1 therapy with pembrolizumab in NSCLC when these tumors had higher 
nonsynonymous mutation burden (6). Thus, identification and modulation of the key immunoregulatory molecules within the tumor microenvironment are warranted to improve patient-tailored multimodal treatment options for NSCLC.

We and others previously demonstrated that molecular alterations commonly found in NSCLC have distinct effects on the therapeutic response to antineoplastic treatment regimens (7-10). The recent study by Skoulidis and colleagues demonstrated that co-occurring genetic alterations in serine/threonine kinase 11/ liver kinase B1 (STK11/LKB1), TP53, and CDKN2A/B (cyclin-dependent kinase inhibitor 2A/B) characterize 3 major subgroups of $K R A S$-mutant lung adenocarcinoma (10). KRAS/TP53 tumors were associated with higher levels of somatic mutations, inflammatory markers, and immune checkpoint effector molecules and improved relapse-free survival. However, KRAS/LKB1 tumors were biologically and therapeutically different: prominent aspects of these tumors were high rates of Kelch-like ECH-associated protein 1-inactivating (KEAP1-inactivating) mutations and low levels of immune markers. An analysis exerted by Schabath et al. on 442 tumors of lung adenocarcinoma patients confirmed the differential biological association of STK11 and TP53 in KRAS-mutant tumors (11). In line with these findings, our own preclinical analysis revealed that loss of the tumor suppressor Stk11/Lkb1 in Kras-mutated tumors induced marked changes within the tumor immune microenvironment in comparison to Kras-mutated tumors specified by accumulation of tumor-associated neutrophils, increased expression of T cell exhaustion markers, and secretion of tumor-promoting cytokines (12). Furthermore, these tumors were not responsive to $\alpha \mathrm{PD}-1$ blockade. Therefore, we speculate that similar genotype-dependent differences may affect therapeutic sensitivity to combinatorial treatment regimens of RT and immunotherapies and might require thorough testing for identification of optimal immune-modulating agents. In order to systematically address this question, we utilized our recently developed preclinical platform for image-guided radiotherapy with two different genetically engineered mouse models (GEMMs) of Kras-driven tumorigenesis, as these allow for autochthonous tumor formation surrounded by a normal tissue microenvironment in an immunocompetent animal (8).

In early-stage disease, as an alternative to surgery, RT is delivered using a focused, targeted approach (stereotactic radiation), sparing vulnerable healthy tissue and draining lymph nodes. This is of critical importance, as the latter are essential in mounting an effective immune response, but only if left functional after RT (13). Thus, despite the local application of stereotactic radiosurgery, the associated immunological effects are elicited systemically and might act on distant metastasis, leading to a phenomenon called "the abscopal effect" (14). Indeed, case reports have demonstrated spontaneous regression of NSCLC metastases outside of the irradiated field (primary adenocarcinoma in the right lower lobe) 12 months after completion of stereotactic body radiotherapy. Although the clinical case presented by Golden et al. describes similar findings in a patient with metastatic NSCLC, it remains unclear whether the additional application of ipilimumab, a cytotoxic T-lymphocyte-associated protein 4 antibody ( $\alpha$ CTLA-4, also known as CD152), augments the antitumor immunity (15). Interestingly, only after application of local RT and subsequent maintenance therapy with ipilimumab, a melanoma patient with prior disease progression on ipilimumab treatment demonstrated a remarkable tumor reduction, most likely mediated by an abscopal effect $(16,17)$.

In this study, we demonstrate that RT leads to an adaptive upregulation of tumor cell PD-L1 expression and concomitant administration of $\alpha \mathrm{PD}-1$ antibody generates effective antitumor immunity and long-term tumor control in Kras-driven murine NSCLC (mNSCLC). Importantly, we provide further evidence that $\alpha \mathrm{PD}-1$ therapy may be best used with radiation in the primary setting, as RT-recurrent tumors do not respond to $\alpha \mathrm{PD}-1$ treatment. We also observe that the underlying molecular alterations markedly influence the treatment response, as Kras/Lkb1-mutated tumors are minimally affected by the combination therapy.

\section{Results}

Synergistic efficacy of RT and PD-1 blockade in Kras-driven $m$ NSCLC. To evaluate the therapeutic efficacy of RT and $\alpha \mathrm{PD}-1$ treatment, we utilized our recently developed preclinical platform for image-guided radiotherapy with lung cancer-relevant GEMMs (8). Application of precise targeted RT is of particular significance to avoid the impact of RT-induced lymphocyte killing or the exacerbation of out-of-field inflammation. In this study, we utilized our previously tested RT regimen, which consists of 17 Gy in 2 fractions of 8.5 Gy, given on 2 consecutive days approximately 24 hours apart $(8.5 \mathrm{~Gy} \times 2)$. While the translation of radiation doses and fractionation schemes used in humans to mice is difficult and not very well studied $(8,18)$, prior work has shown that multi-fraction RT is more effective than single-fraction RT when combined with CTLA-4 checkpoint blockade in syngeneic tumor models (19). Additionally, we used a previously described 
timing approach of the immunomodulatory agent and initiated $\alpha \mathrm{PD}-1$ treatment 6 hours after the second fraction of 8.5 Gy (Figure 1A) (20). After RT and continuous $\alpha$ PD-1 treatment, we measured the tumor growth kinetics every 2 weeks using noninvasive MRI. Strikingly, Kras mice treated with the combination therapy showed significant tumor regression of the target lesion 4 weeks after treatment initiation and remained stable with very low tumor burden after completion of 12 weeks (Figure 1B). Detailed analysis of the tumor growth curves of 10 individually treated Kras mice revealed the existence of a commonly observed phenomenon in radiographic assessment of therapeutic efficacy of immunotherapy (Figure 1C): tumor response (determined by conventional tumor response criteria such as Response Evaluation Criteria in Solid Tumors [RECIST] and World Health Organization criteria) may be seen after an initial increase in tumor burden or during/after the presentation of new lesions (21-23). The biologic anticancer mechanisms of immunotherapeutic agents induce activation and proliferation of $\mathrm{T}$ cells and lead to tumor infiltration by $\mathrm{T}$ cells $(21,24,25)$. In consequence, patients treated with immunotherapy show unique imaging manifestations, which require accurate radiological interpretation.

In our cohort of dual-treated mice, we found that 7 of 10 mice reached a partial response (at least $30 \%$ volume reduction of the target lesion) and 3 of these responders even showed tumor shrinkage of $65 \%-70 \%$ compared with the baseline tumor volume (Figure 1C). It is important to note that these antitumor responses remained for a period of 12-17 weeks. Despite these encouraging results, we were unable to perform further tumor measurements of the targeted lesion, as contralateral tumor growth was observed in 6 responding mice, likely due to spillage of Adeno-Cre virus in the contralateral lung (Supplemental Figure 1A; supplemental material available online with this article; doi:10.1172/jci.insight.87415DS1). However, dual-treated tumors in the left lung lobe remained small at time of discontinuation. Although 3 of 10 mice relapsed after dual therapy with RT and $\alpha$ PD-1, 2 of these mice also had a pronounced tumor volume reduction (roughly $40 \%-50 \%$ ), which lasted for 12 weeks. When comparing the treatment responses of dual-treated Kras tumors to unirradiated, RT-, and $\alpha$ PD-1-treated cohorts, the dual therapy yielded significant superiority over the single RT arm by 10 weeks after treatment initiation $(P=0.0097$, ANOVA; Figure 1D). The supremacy of the dual treatment further translated into a significant survival benefit when compared with unirradiated and $\alpha \mathrm{PD}-1$-treated mice $(P=0.0032$ and $P=0.0013$, respectively, log-rank test, Bonferroni-corrected threshold 0.0167; Figure 1E). Due to the technical limitations of our animal model and subsequent death of other cause (spillage of Adeno-Cre to the right lung), the dual treatment did not reach significance over the single RT arm but showed that there is a positive trend of survival benefit (Figure 1E). To account for this limitation, we calculated progression-free survival and found a significant difference between dual-treated mice and unirradiated and RT-treated mice $(P=0.0002$, and $P=0.0045$, respectively, log-rank test, Bonferroni-corrected threshold 0.0167; Figure $1 \mathrm{~F}$ ). In summary, we demonstrated that RT and PD-1 blockade promoted significant and durable antitumor efficacy in Krasdriven mNSCLC, whereas the single treatments only induced stable disease for a shorter time period. $90 \%$ of the dual-treated mice demonstrated $30 \%-70 \%$ tumor volume reduction compared with the baseline tumor volume, which persisted for 12 to 17 weeks. Single treatment with either RT or PD-1 induced no tumor shrinkage, but tumor volume measurements remained stable for 8 and 10 weeks, respectively.

Radiobiologic effects on the immune tumor microenvironment. We next sought to determine how RT affects the quantity and subsequent recruitment of tumor-associated immune cell populations. Due to the limited literature on leucocyte behavior elicited by RT and in comparable models, we analyzed the immune cell infiltrates at two time points after RT, 24 and 96 hours (Figure 2A). Using flow cytometry, we analyzed the total numbers of tumor-infiltrating lymphoid (Figure 2B, top) and myeloid cell populations (Figure 2B, bottom) before and after RT. We detected a significant decline in all lymphoid populations 24 hours after RT, indicating their radiosensitivity (total T cells $P<0.0001, \mathrm{CD}^{+} \mathrm{T}$ cells $P=0.0004, \mathrm{CD}^{+} \mathrm{T}$ cells $P<0.0001$, B cells $P=0.0001$, NK cells $P=0.0147$; Figure $2 \mathrm{~B}$, top, and Figure 2C). Investigation of cell counts at 96 hours after RT showed that B, NK, and T cell subpopulations began to increase, albeit only NK cells were no longer significantly diminished compared with values from naive tumors. Thus, despite high doses of RT, all analyzed lymphoid populations are capable of repopulating the tumor microenvironment 96 hours after RT. Several clinical reports suggest that a high density of tumor-infiltrating lymphocytes is associated with favorable overall prognosis in patients with cancer (26-28) and that lower percentages of Tregs correlate with improved outcomes (29, 30). Analysis of the Tregs in Kras tumors after RT showed a $65 \%$ decrease in population, which remained partially stable after 3 days (unirradiated vs. 24 hours $P<0.0001$, unirradiated vs. 96 hours $P<0.0001$; Figure 2C). As a consequence, we observed that 


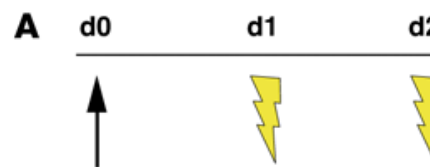

Tumor detection 8.5 Gy (MRI) d2
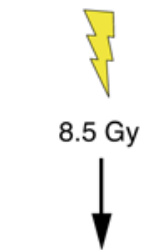

$6 \mathrm{~h}$ later aPD-1 antibody treatment
B

Pre-treatment

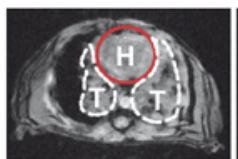

4 weeks post $\mathrm{RT}+\alpha \mathrm{PD}-1$

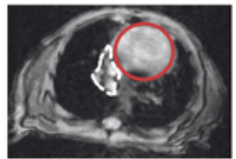

12 weeks post $\mathrm{RT}+\alpha \mathrm{PD}-1$

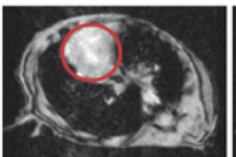

D
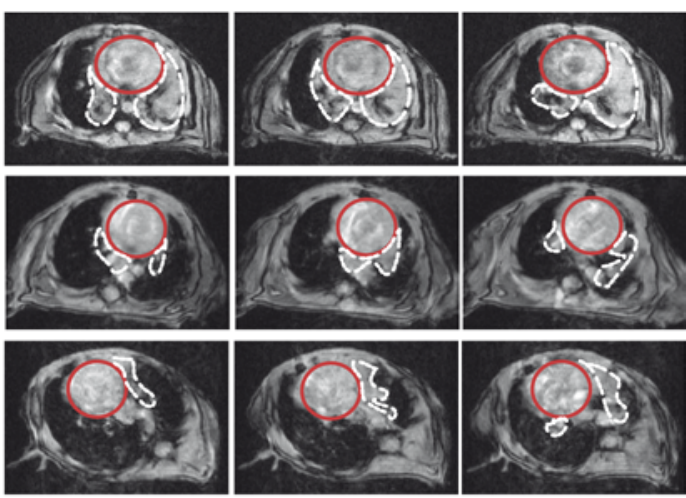

C

$8.5 \mathrm{~Gy} \times 2+\alpha \mathrm{PD}-1$ antibody treatment response
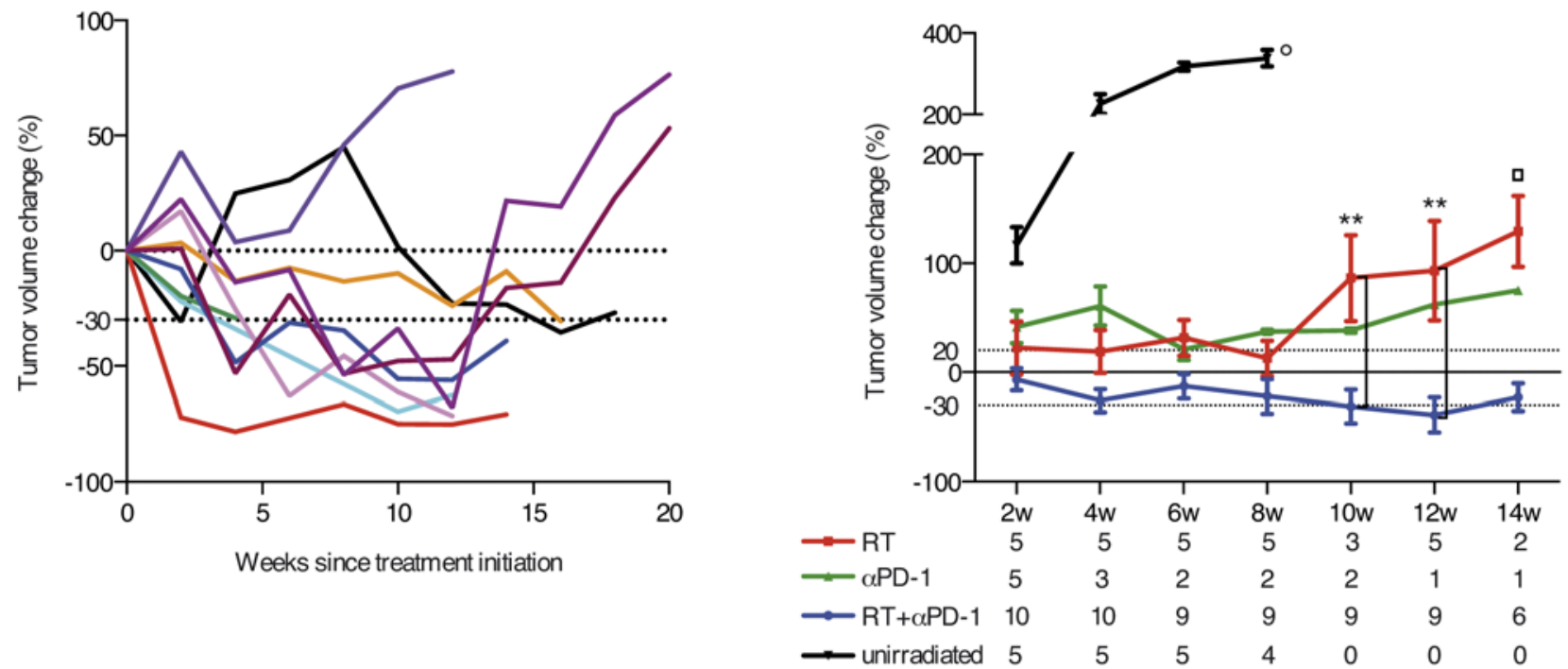

E

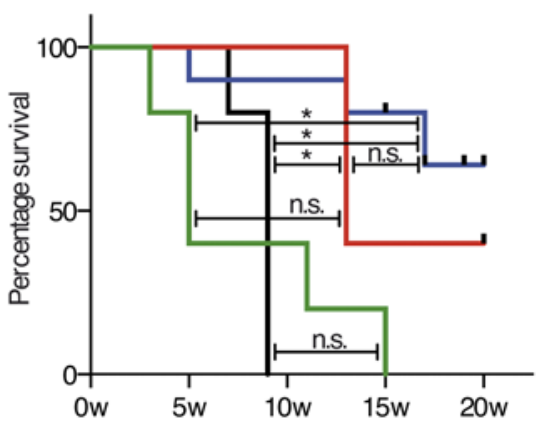

Number at risk

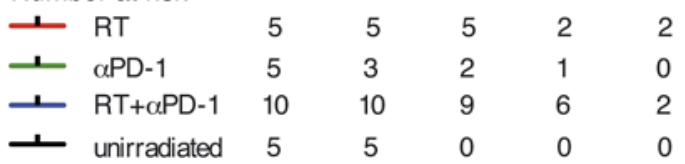

$\mathbf{F}$

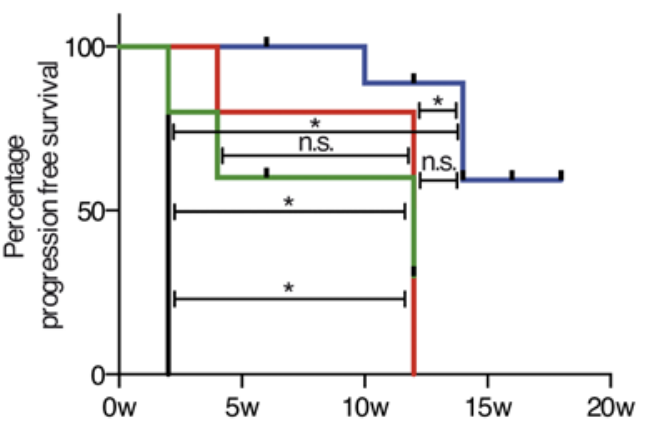

Number at risk

\begin{tabular}{|c|c|c|c|c|c|}
\hline 1 & RT & 5 & 4 & 4 & 0 \\
\hline 1 & $\alpha \mathrm{PD}-1$ & 5 & 3 & 2 & 0 \\
\hline 1 & $\mathrm{RT}+\alpha \mathrm{PD}-1$ & 10 & 10 & 8 & 2 \\
\hline L & unirradiated & 5 & 0 & 0 & 0 \\
\hline
\end{tabular}

Figure 1. Blockade of the PD-1/PD-L1 axis potentiates the antitumor efficacy of RT in Kras-mutant murine NSCLC. (A) Schematic representation of combinatorial treatment schedule. (B) Representative MRI images of a Kras-driven tumor (one row represents 4 mm of lung) treated with RT and PD-1 blockade at different time points (baseline, 4 weeks, and 12 weeks after treatment initiation). H, heart (circled in red); T, tumor (circled with a white dotted line). (C) Tumor volume kinetics after dual treatment with RT and $\alpha \mathrm{PD}-1$. Each line represents one mouse $(n=10)$. (D) Treatment response to RT, $\alpha \mathrm{PD}-1$, or a combination of both is depicted. Data from unirradiated and RT cohort were previously published (8). Numbers below time points indicate amount of mice evaluated per group. (D-F) Data are represented as mean \pm SEM. $P$ values were calculated using 1-way ANOVA with Tukey's multiple comparisons test. ${ }^{* *} P<0.01$. ${ }^{\circ}$ This control group had to be discontinued due to extensive tumor burden or death. As fewer than 3 RT-treated mice were alive at this time point, statistical analysis was omitted. (E) Kaplan-Meier survival analysis of study cohorts presented in $\mathbf{D}$ calculated from treatment initiation. (F) Kaplan-Meier progression-free survival analysis of study cohorts presented in $\mathbf{D}$ calculated from treatment initiation. (E and F) $P$ values were calculated using log-rank test corrected for multiple comparisons. A $P$ value of less than 0.0167 (Bonferroni-corrected threshold) was considered statistically significant. ${ }^{*} P<0.05$. d, days; $w$, weeks; RT, radiation therapy; PD-L1, programmed death-ligand 1; PD-1, programmed cell death 1. 
A

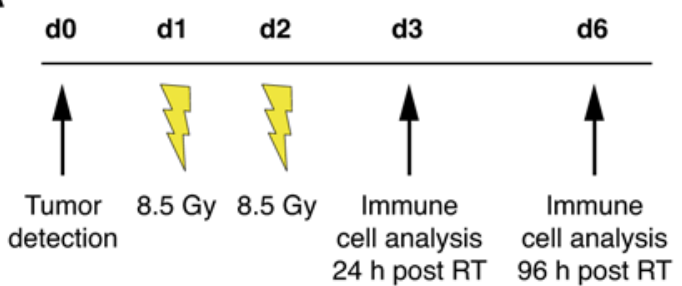

C

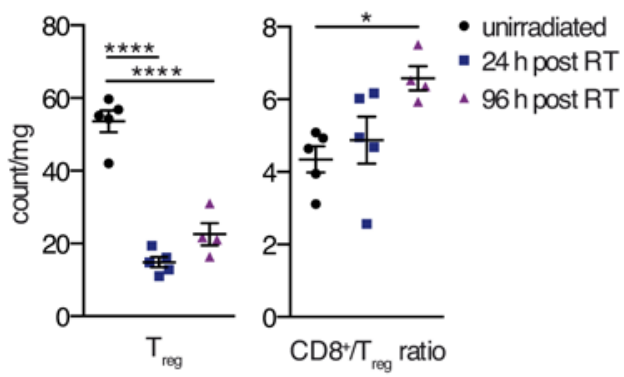

D

EpCAM+ tumor cells unirradiated / $24 \mathrm{~h} / 24 \mathrm{~h}$

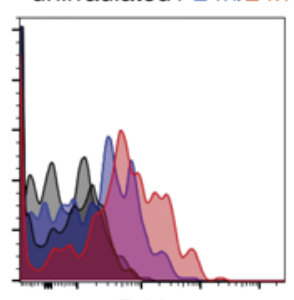

Pd-l1

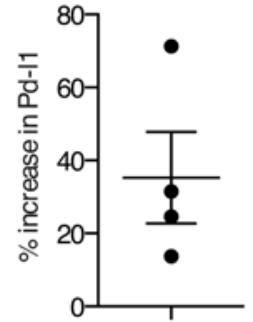

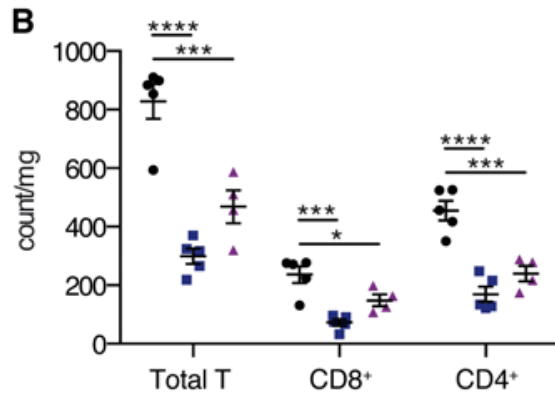
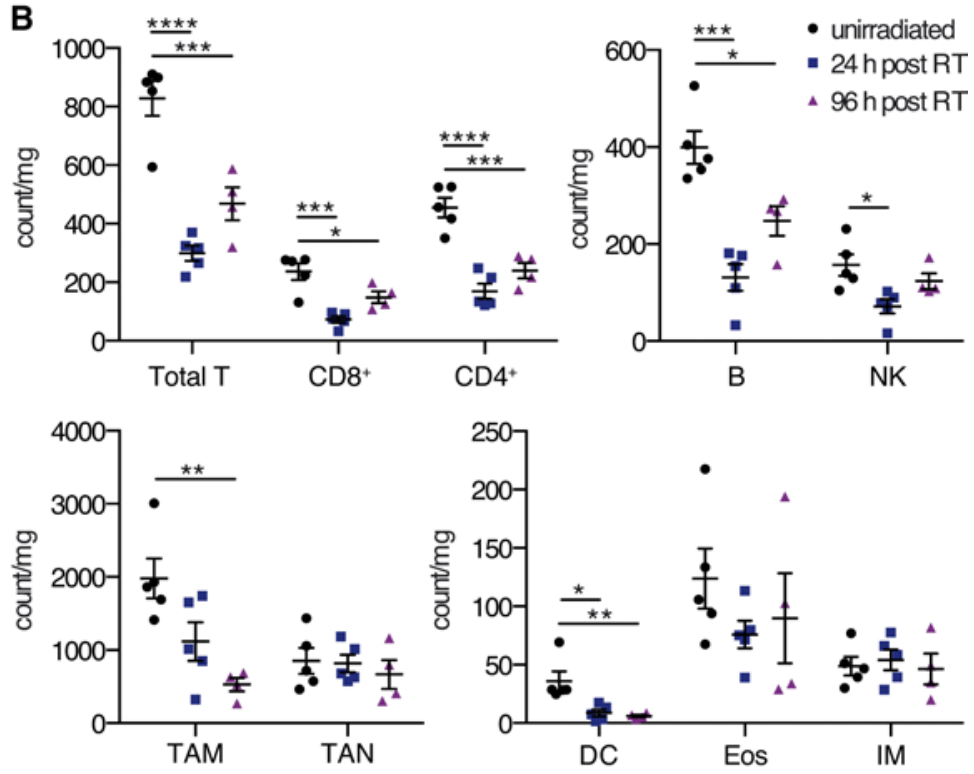

E

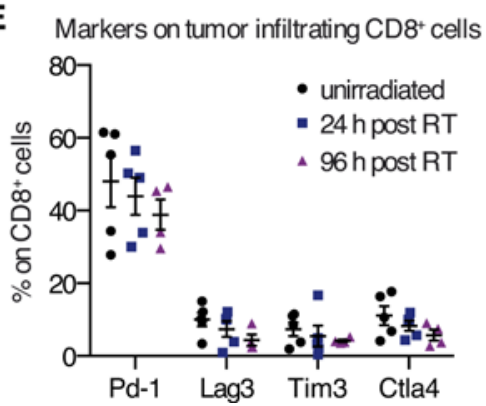

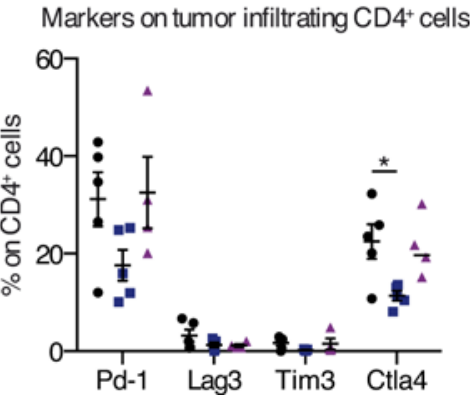

Figure 2. Phenotyping of tumor-associated immune cell populations after RT in Kras-mutant murine NSCLC. (A) Schematic representation of shortterm analysis time points. (B) Representative flow cytometry data (live/single/total CD45+ cells). Total numbers of tumor-infiltrating lymphoid cells (top) and myeloid cells (bottom) of unirradiated tumors or shortly (24 and 96 hours) after RT. (C) Total number of tumor-associated Tregs at indicated time points. CD8 ${ }^{+} /$Treg ratio was calculated from B and C. (D) PD-L1 expression of EpCAM+ tumor cells unirradiated and 24 hours after RT. Each dot represents one mouse $(n=4)$. (E) Expression of inhibitory T cell markers on CD8 $8^{+}$and CD4+ $T$ cells from unirradiated and RT-treated tumors. Representative data are shown with 5 (unirradiated), 5 (24 hours after RT), and 4 ( 96 hours after RT) mice (B, C, and E). Data are represented as mean \pm SEM. $P$ values were calculated using 1-way ANOVA with Tukey's multiple comparisons test. ${ }^{* * *} P<0.0001 ;{ }^{* * *} P<0.001 ;{ }^{* *} P<0.01 ;{ }^{*} P<0.05$. d, days; h, hours; B, B cells; TAM, tumor-associated macrophages; TAN, tumor-associated neutrophils; Eos, eosinophils; IM, inflammatory monocytes; EpCAM, epithelial cell adhesion molecule; Lag3, lymphocyte-activation gene 3; Tim3, T cell immunoglobulin and mucin-domain containing-3; Ctla4, cytotoxic T-lymphocyte-associated protein 4.

the $\mathrm{CD}^{+} /$Treg ratio increased 96 hours after RT. To further demonstrate a beneficial interaction between RT, T cells, and the PD-1/PD-L1 axis, we analyzed PD-L1 expression 24 hours after RT on Kras tumor cells and demonstrated its upregulation (Figure 2D). Furthermore, we showed stable expression of PD-1 on $\mathrm{CD} 4^{+}$and $\mathrm{CD} 8^{+} \mathrm{T}$ cells upon RT (Figure $2 \mathrm{E}$ ).

Myeloid cells, such as macrophages, neutrophils, DCs, eosinophils, and monocytes, are considered to be more radioresistant in contrast to lymphoid cells $(31,32)$. Our present study partly confirms these findings (Figure 2B, bottom). Tumor-associated neutrophils (TAN; CD11b+Ly-6G+), eosinophils (CD11b $\mathrm{b}^{+} \mathrm{Ly}-6 \mathrm{G}^{-}$ Siglec $\left.\mathrm{F}^{+} \mathrm{CCR} 3^{+}\right)$, and inflammatory monocytes $\left(\mathrm{CD} 11 \mathrm{~b}^{+}\right.$Ly- $6 \mathrm{G}^{-}$SiglecF-Ly- $\left.6 \mathrm{C}^{\text {hi }}\right)$ showed no marked differences in number 24 and 96 hours after RT. In contrast, tumor-associated macrophages (TAM; CD11 $\mathrm{c}^{+} \mathrm{CD}$ $\left.11 b^{-} \mathrm{CD}^{-} 103^{-}\right)$and $\mathrm{CD} 103^{+}$DCs displayed a significant decline after two fractions of RT.

Taken together, our data thus suggest that RT induces favorable changes within the tumor microenvironment - augmented $\mathrm{CD} 8^{+} /$Treg ratio, rise in PD-L1 expression on Kras tumor cells, and unchanged expression of PD-1 on tumor-associated T cells - for additional blockade of the PD-1/PD-L1 axis.

Immune phenotyping of Kras tumors progressed after RT. The promising antineoplastic synergy of concurrent RT and $\alpha \mathrm{PD}-1$ treatment in mNSCLC led us to the question whether RT-relapsed Kras tumors 
A

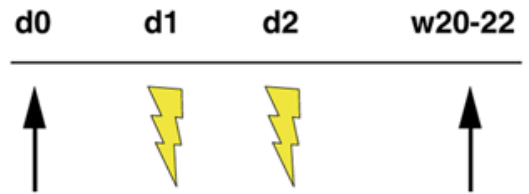

Tumor 8.5 Gy 8.5 Gy detection

C

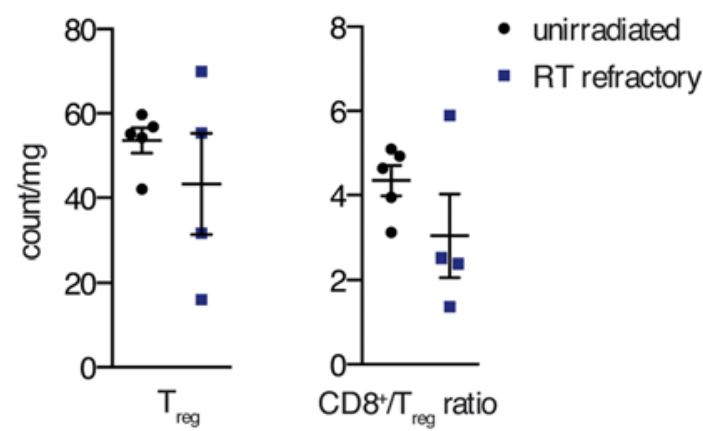

D

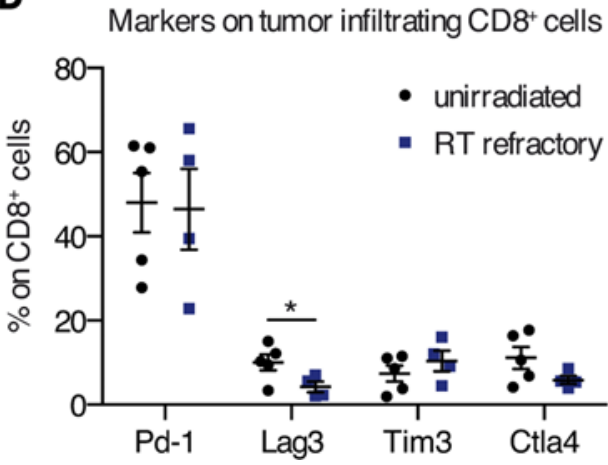

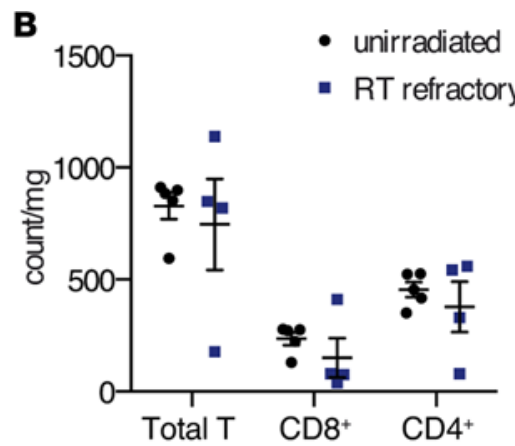
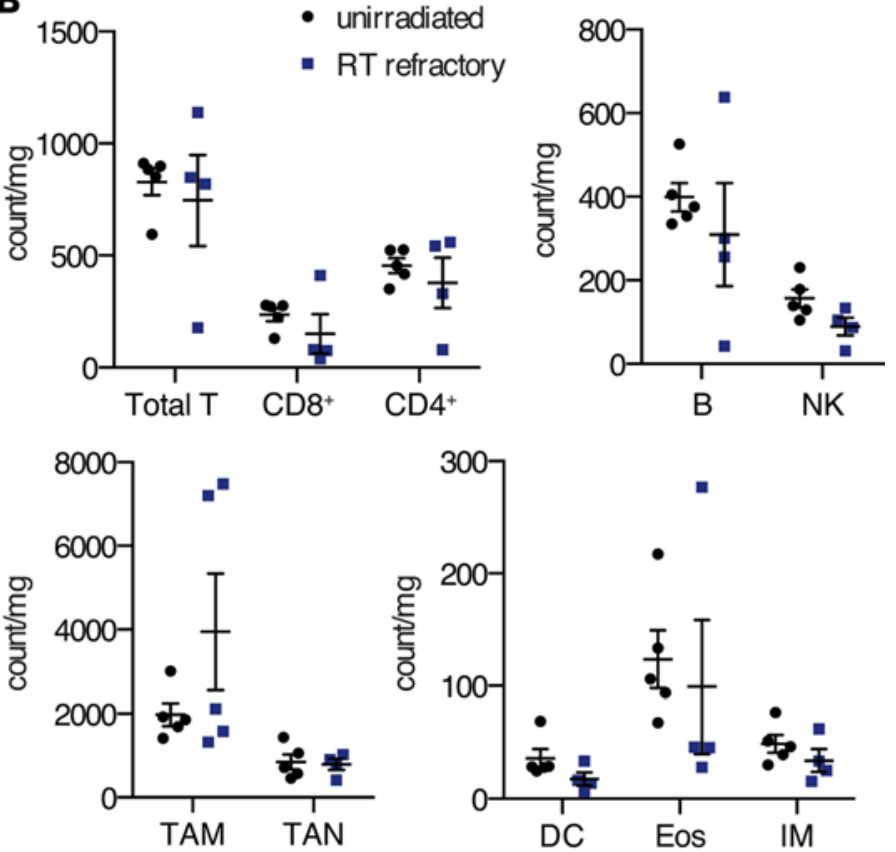

\section{Markers on tumor infiltrating $\mathrm{CD} 4^{+}$cells}

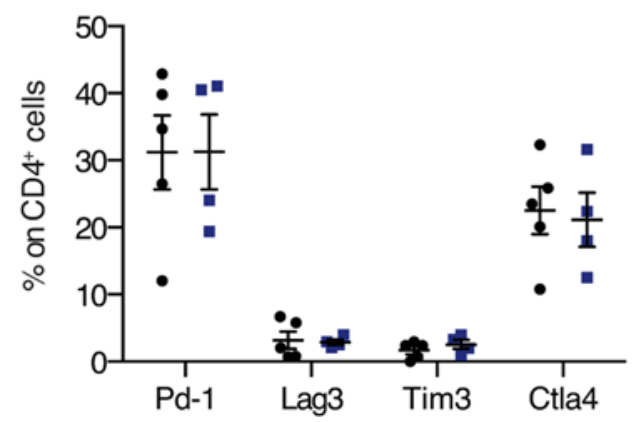

Figure 3. Phenotyping of tumor-associated immune cell populations in RT-refractory Kras-mutant murine NSCLC. (A) Schematic representation of analysis time points after RT. (B) Representative flow cytometry data (live/single/total CD45+ cells). Total numbers of tumor-infiltrating lymphoid cells (top) and myeloid cells (bottom) of unirradiated and RT-relapsed tumors (at 20 to 22 weeks after RT). (C) Total number of tumor-associated Tregs from unirradiated and RT-relapsed tumors. CD8 ${ }^{+} /$Treg ratio was calculated from $\mathbf{B}$ and $\mathbf{C}$. (D) Expression of inhibitory T cell markers on CD8 ${ }^{+}$and CD4 ${ }^{+} \mathrm{T}$ cells from unirradiated and RT-relapsed tumors. Representative data are shown conducted with 5 unirradiated and 4 RT-treated mice (same cohort as described in Figure 2) (B-D). Data are represented as mean \pm SEM. $P$ values were calculated using 2-tailed Student's $t$ test. ${ }^{*} P<0.05$. d, days; w, weeks; RT, radiation therapy; PD-1, programmed cell death 1; TAM, tumor-associated macrophages; TAN, tumor-associated neutrophils; Lag3, lymphocyte-activation gene 3; Tim3, T cell immunoglobulin and mucin-domain containing-3; Ctla4, cytotoxic T-lymphocyte-associated protein 4.

would also show treatment response to PD-1 blockade and thereby offer another therapeutic option for patients with recurrent NSCLC. In a first attempt to address this question, we examined the tumor-associated immune cell infiltrates $20-22$ weeks after RT when mice were close to reaching euthanasia criteria (the cohort of aged mice is described in the legend for Figure 2) (Figure 3). As unirradiated Kras mice only survive up to 8 weeks after tumor identification, we analyzed the immune cell populations at this time point. The cell counts of all immune populations were slightly reduced compared with those of unirradiated Kras tumors, aside from TAM. Although several preclinical and clinical reports have demonstrated that RT is associated with subsequent recruitment of primarily M2 TAM (33-37), we only found increased numbers of TAM in half of the tumors. M2 macrophages, in contrast to the M1 phenotype, have been shown to promote tumor growth and suppress antitumor immunity, especially towards $\mathrm{T}$ cells (38). Detailed analysis of the T cell compartment in all RT-refractory tumor samples showed a small reduction in Tregs and an accompanying $\mathrm{CD} 8^{+} /$Treg ratio drop due to a concomitant diminution of $\mathrm{CD}^{+} \mathrm{T}$ cells (Figure $3 \mathrm{C}$ ). Besides a decreased expression of lymphocyte-activation gene 3 (Lag3, also 
A

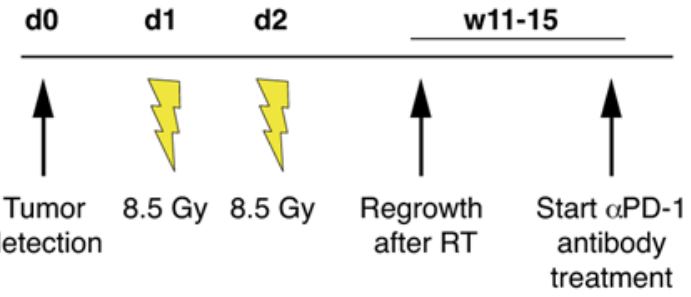

B Treatment response to $\alpha$ PD-1 in RT-refractory Kras tumors
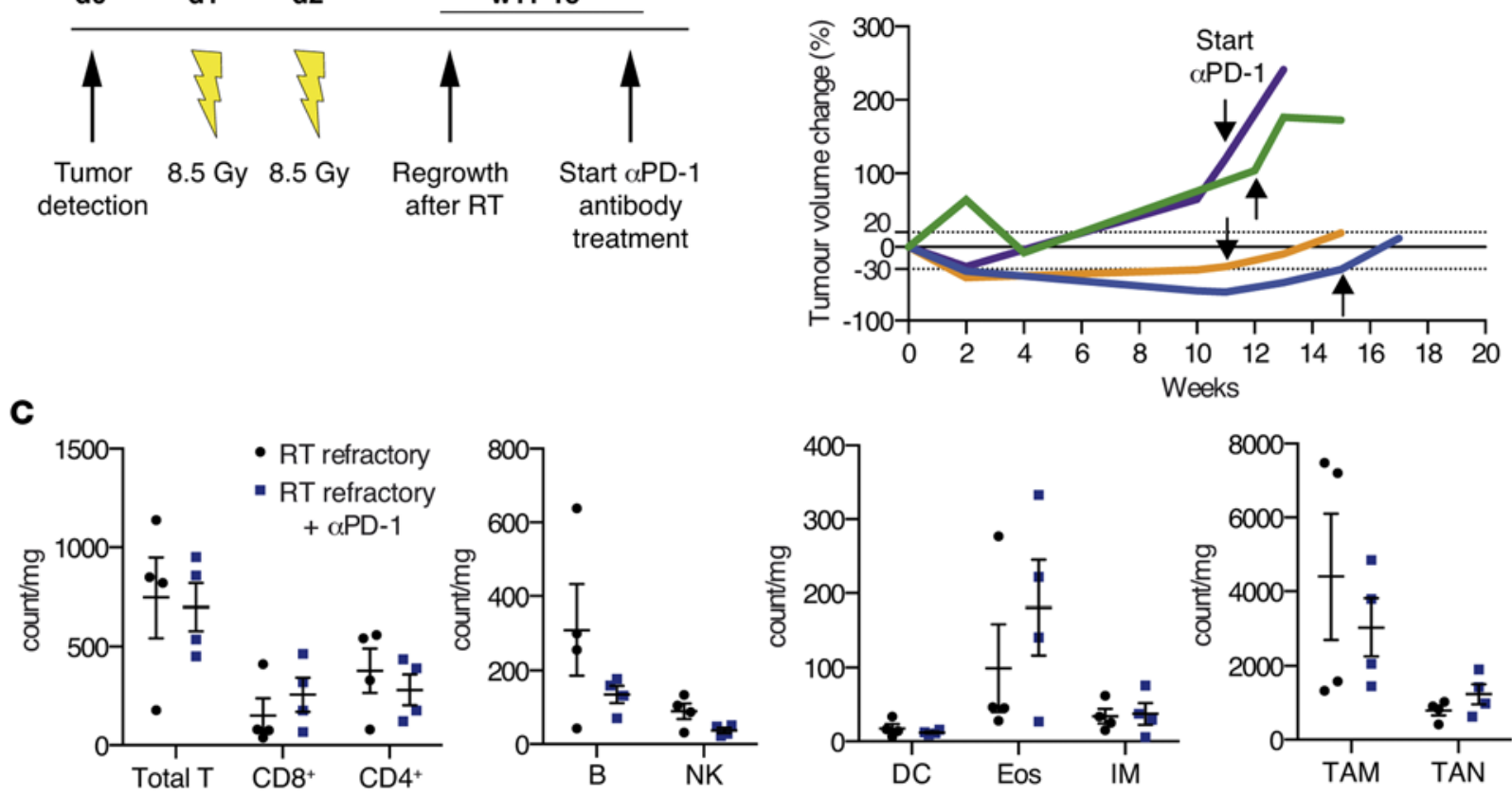

D
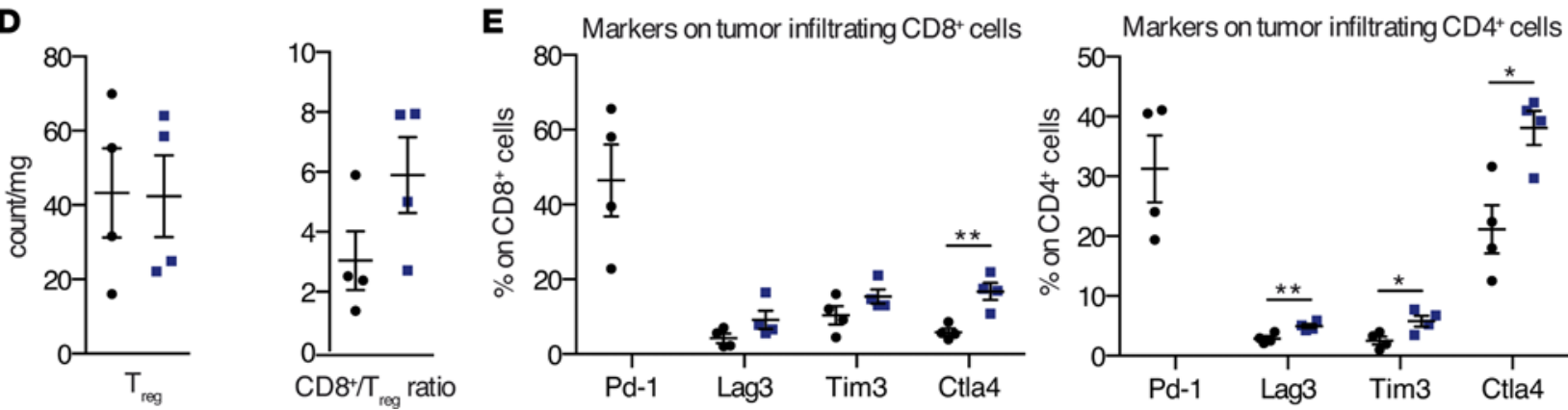

Figure 4. Phenotyping of tumor-associated immune cell populations in RT-refractory $\alpha$ PD-1-treated Kras-mutant murine NSCLC. (A) Schematic representation of treatment schedule. $\alpha$ PD-1 treatment was initiated once tumor recurrence was detected (11-15 weeks after RT). (B) Tumor volume kinetics of RT-relapsed tumors treated with $\alpha \mathrm{PD}-1$. Each line represents one mouse $(n=4)$. Arrows indicate the beginning of $\alpha \mathrm{PD}-1$ treatment. (C) Representative flow cytometry data (live/single/total CD45+ cells). Total numbers of tumor-infiltrating lymphoid cells and myeloid cells of RT-refractory or RT-refractory $\alpha$ PD-1treated tumors. (D) Total number of tumor-associated Tregs at indicated time points. CD8 ${ }^{+} /$Treg ratio was calculated from $\mathbf{C}$ and $\mathbf{D}$. (E) Expression of inhibitory T cell markers on $\mathrm{CD}^{+}$and $\mathrm{CD}^{+}{ }^{+} \mathrm{T}$ cells of RT-recurrent and RT-recurrent $\alpha \mathrm{PD}-1$-treated tumors. Representative data are shown conducted with $4 \mathrm{RT}$-relapsed and 4 RT-relapsed $\alpha$ PD-1-treated mice (C-E). Data are represented as mean \pm SEM. $P$ values were calculated using 2-tailed Student's $t$ test. ${ }^{* *} P<0.01 ;{ }^{*} P<$ 0.05. d, days; w, weeks; RT, radiation therapy; PD-1, programmed cell death 1; TAM, tumor-associated macrophages; TAN, tumor-associated neutrophils; Lag3, lymphocyte-activation gene 3; Tim3, T cell immunoglobulin and mucin-domain containing-3; Ctla4, cytotoxic T-lymphocyte-associated protein 4.

know as $\mathrm{CD} 223$ ) on $\mathrm{CD} 8^{+} \mathrm{T}$ cells, further inhibitory $\mathrm{T}$ cell markers on $\mathrm{CD} 4^{+}$and $\mathrm{CD} 8^{+} \mathrm{T}$ cells did not change significantly in these RT-recurrent tumors (Figure 3D). In accordance with these findings, we further observed that the correlation of $\mathrm{TAM}$ and the $\mathrm{CD} 8^{+} / \mathrm{CD} 4^{+}$ratio significantly changed between the unirradiated and RT-refractory treatment group: in unirradiated tumors there was a strong positive correlation between the two variables $(r=0.74, P=0.0935)$ and a very strong negative correlation in RT-refractory tumors $(r=-0.85, P=0.069)(P=0.016$, test for difference of 2 independent correlation coefficients; Supplemental Table 2). Similar significant changes were found for the association between neutrophils and B cells $(P=0.0001)$ and neutrophils and eosinophils $(P=0.0054$, test for difference of 2 independent correlation coefficients; Supplemental Table 1), respectively.

Given our observation that PD-1 expression on $\mathrm{CD}^{+}$and $\mathrm{CD} 8^{+} \mathrm{T}$ cells remains constant in RT-naive and RT-relapsed tumors, we expected that the latter might respond to PD-1 blockade.

$R T$-refractory Kras tumors do not respond to PD-1 blockade treatment. In order to investigate whether progressed Kras tumors after RT respond to $\alpha \mathrm{PD}-1$ treatment, we designed another preclinical trial. Using non- 
A

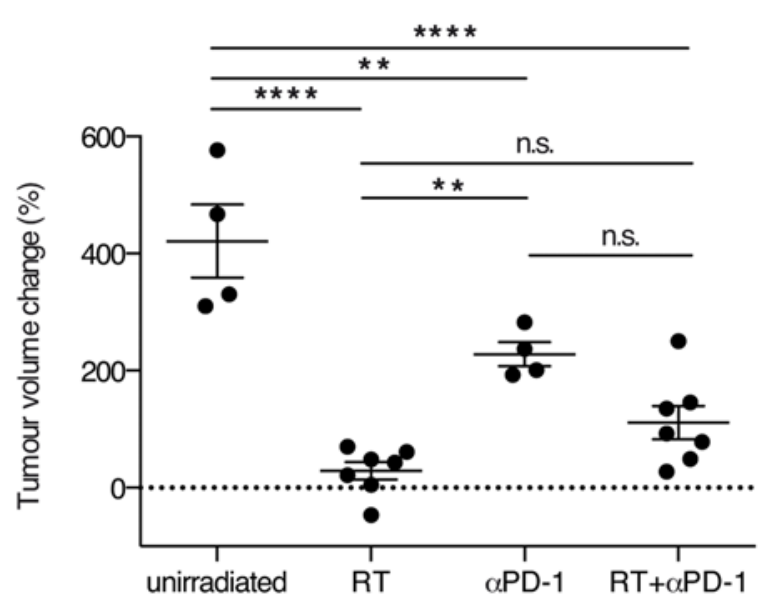

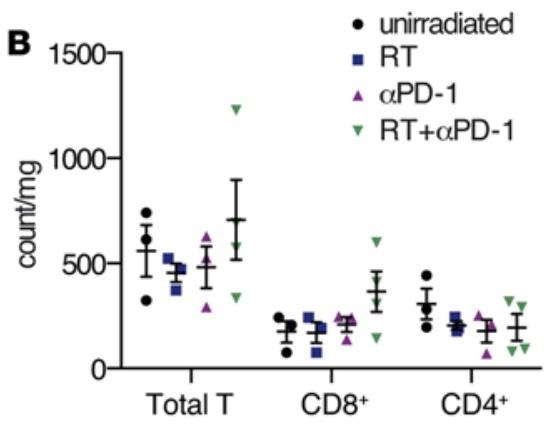
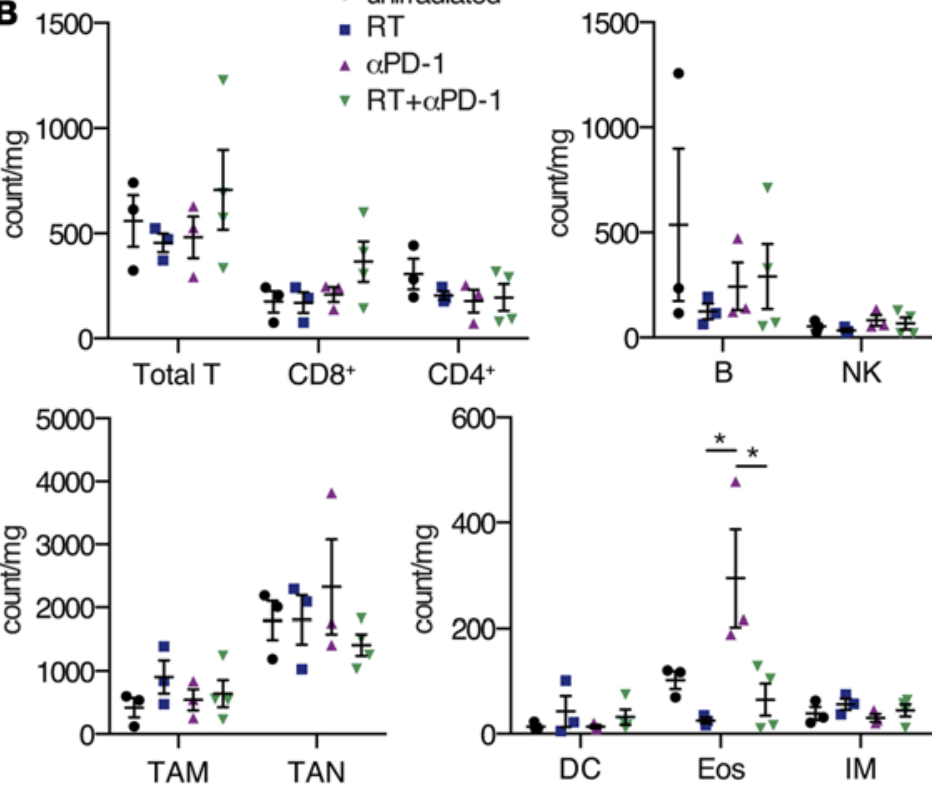

D Markers on tumor infiltrating $\mathrm{CD} 8^{+}$cells
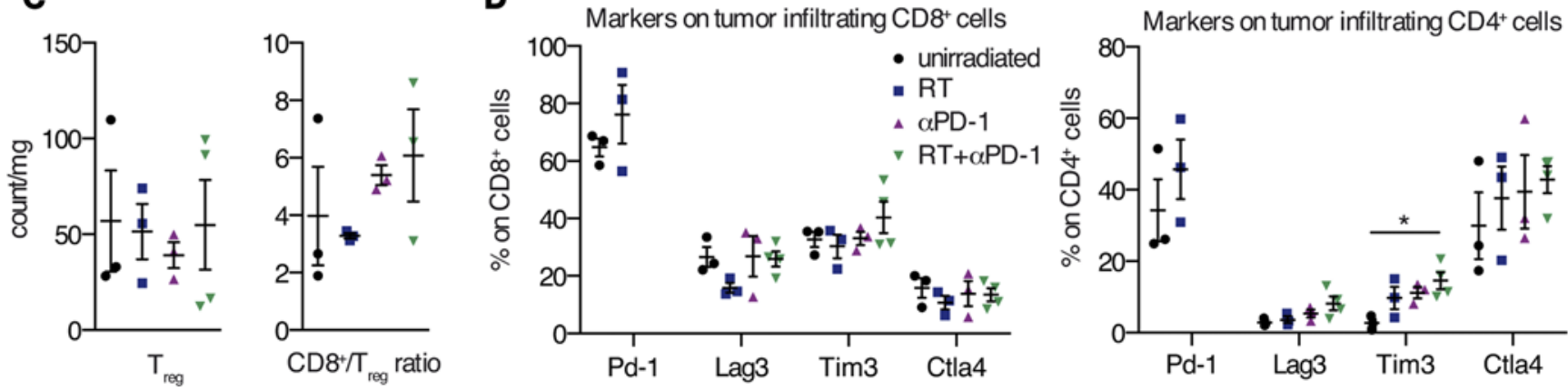

Figure 5. Phenotyping of tumor-associated immune cell populations after RT, $\alpha$ PD-1, and combination therapy in Kras/Lkb1-mutant murine NSCLC. (A) Treatment response of unirradiated $(n=4)$ and RT- $(n=7), \alpha$ PD-1- $(n=4)$, and RT $+\alpha$ PD-1-treated $(n=7)$ mice at 2 weeks. Each dot represents one mouse. Data of the RT cohort was partly published previously (8). (B) Representative flow cytometry data (live/single/total CD45+ cells). Total numbers of tumor-infiltrating lymphoid cells and myeloid cells of tumors from indicated treatment groups. (C) Total number of tumor-associated Tregs of indicated treatment groups. CD8 $8^{+} /$Treg ratio was calculated from $\mathbf{B}$ and $\mathbf{C}$. (D) Expression of inhibitory T cell markers on CD $8^{+}$and $C D 4^{+} T$ cells of indicated treatment groups. PD-1 expression could not be measured in $\alpha$ PD-1-treated tumors due to antibody binding. Representative data are shown conducted with 3 unirradiated and 3 RT-treated, $3 \alpha \mathrm{PD}$-1-treated, and 4 dual-treated mice (B-D). Data are represented as mean \pm SEM. $P$ values were calculated using 1-way ANOVA with Tukey's multiple comparisons test. ${ }^{* * *} P<0.0001 ;{ }^{* *} P<0.01 ;{ }^{*} P<0.05$. RT, radiation therapy; PD-1, programmed cell death 1; TAM, tumor-associated macrophages; TAN, tumor-associated neutrophils; Lag3, lymphocyte-activation gene 3; Tim3, T cell immunoglobulin and mucin-domain containing-3; Ctla4, cytotoxic T-lymphocyte-associated protein 4

invasive MRI, we determined the time point of tumor regrowth after RT to initiate $\alpha$ PD-1 therapy (Figure $4 \mathrm{~A})$. However, none of the mice with recurrent tumors after RT responded to $\alpha$ PD-1 antibody treatment; instead, they showed continuous tumor growth (Figure 4B). Subsequent examination of tumor-associated immune cell populations in RT-relapsed tumors treated with $\alpha \mathrm{PD}-1$ revealed a marked reduction of $\mathrm{B}$ cells, NK cells, and CD103+ DCs compared with RT-recurrent tumors without $\alpha$ PD-1 treatment (Figure $4 \mathrm{C}$ ). Addition of $\alpha \mathrm{PD}-1$ also decreased the number of TAM and mediated a small increase in TAN. Additionally, we observed an influx of $\mathrm{CD} 8^{+} \mathrm{T}$ cells, promoting an improved, albeit nonsignificant, $\mathrm{CD} 8^{+} /$Treg ratio (Figure 4D). Surprisingly, the levels of the investigated inhibitory T cell markers Lag3, T cell immunoglobulin mucin-domain containing-3 (Tim3, also known as hepatitis A virus cellular receptor 2 [Havcr2]), and CTLA- 4 were significantly elevated, especially on CD4 ${ }^{+} \mathrm{T}$ cells in the group of RT-refractory $\alpha \mathrm{PD}-1-$ treated mice (Lag3 $P=0.0097$, Tim3 $P=0.0285$, CTLA-4 $P=0.0140$; Figure 4E). Concordantly, we found that genes associated with $\mathrm{T}$ cell exhaustion and inhibition trended to be more highly expressed in the RT-recurrent group treated with $\alpha$ PD-1 (NanoString nCounter analysis, Supplemental Figure 2A). Given this observation, we speculated whether this tumorigenic $\mathrm{T}$ cell suppression was potentially mediated by 
M2 macrophages as previously shown (38). Hence, we interrogated our data set of murine cancer immune genes for M1- and M2-associated genes but observed no significant trends (Supplemental Figure 2B).

In conclusion, our data suggest that $\alpha \mathrm{PD}-1$ application in RT-relapsed Kras tumors is not sufficient to stop tumor growth but induces increased expression of inhibitory T cell markers upon treatment.

$R T$ and $\alpha P D-1$ treatment are less effective in Kras/Lkb1-mutant NSCLC. We next sought to determine whether the synergistic therapeutic effect of RT and PD-1 blockade would yield a similar tumor response in Kras-driven mNSCLC, with concomitant loss of the tumor suppressor Lkb1 (also known as Stk11). Inactivating mutations of $S T K 11 / L K B 1$ are found in up to $30 \%$ of $K R A S$-mutated lung adenocarcinomas (3941). Preclinical and clinical studies demonstrate that $K R A S / L K B 1$-mutant neoplasms are more aggressive resulting in enhanced metastasis and reduced survival (41-43). Furthermore, we reported that the drug sensitivity of Kras-driven mNSCLC is dramatically impacted depending on the additional loss of either $L k b 1$ or Trp53 (7). More recently, we observed that Lkb1 inactivation in mNSCLC causes significant changes within the tumor microenvironment by mediating accumulation of TAN and reduction of tumor-infiltrating lymphocytes, increased expression of $\mathrm{T}$ cell inhibitory markers, and secretion of tumor-promoting cytokines (12). Additionally, we found that $L k b 1$ alteration is negatively correlated with PD-L1 expression on murine and human tumor cells. However, modulation of the inflammatory microenvironment by either IL-6-neutralizing antibody or antibody-mediated neutrophil depletion reverted the immune-associated phenotype and had therapeutic efficacy. Hence, we speculated that RT might exert similar immunomodulatory effects on the Lkb1-deficient tumor microenvironment. In comparison to Kras mice, we previously observed that Kras/Lkb1 mice have more rapid tumor growth kinetics when evaluated in our single lung nodule model (8). Despite careful intrathoracic application of Adeno-Cre and close clinical monitoring, we were unable to follow treatment response in these mice for longer periods due to rapid tumor growth. Mice treated with either RT or $\alpha \mathrm{PD}-1$ alone recapitulated our previously reported therapeutic responses and demonstrated significant inhibition or slowed tumor enlargement compared with naive tumors, respectively $(8,12)$. The antitumor efficacy of RT was significantly better than PD-1 blockade in Kras/Lkb1 tumors. However, application of dual therapy was not synergistic (Figure 5A). To exclude the possibility that this lack of tumor shrinkage may be masked by influx of recruited immune cells leading to a "pseudo-progression," we correlated the absolute numbers of tumor versus immune cells per milligram tumor among the treatment cohorts and observed similar increases of immune cell numbers (Supplemental Figure 4A). A small number of Kras/Lkb1 mice treated with the combination therapy survived beyond 4 weeks of study, and we were able to document a massive tumor volume increase accompanied by pleural effusion (Supplemental Figure 4B).

To understand the poor efficacy in Kras/Lkb1 tumors, we performed immune panel analysis on these tumors to identify the pharmacodynamic changes upon treatment. Although we found that the highest degree of usually considered favorable antitumor changes within the $\mathrm{T}$ cell compartment was detected upon combinatorial treatment (Figure 5B), these changes did not reach significance. Predominantly, the counts of $\mathrm{CD}^{+}$and $\mathrm{CD}^{+} \mathrm{T}$ cells switched, leading to a more beneficial $\mathrm{CD} 8^{+} /$Treg ratio (Figure $5 \mathrm{C}$ ). Albeit the expression of $\mathrm{T}$ cell inhibitory markers on $\mathrm{CD} 8^{+} \mathrm{T}$ cells was not significantly altered among the treatment groups, our data showed an increase in Lag3, Tim3, and CTLA-4 on CD4 ${ }^{+} \mathrm{T}$ cells when comparing untreated and dual-treated Kras/Lkb1 tumors (Figure 5D). Moreover, we were surprised to find a marked influx of tumor-homing eosinophils in Kras/Lkb1 tumors treated with $\alpha \mathrm{PD}-1$ (Figure 2B). This was consistent with a recent analysis by Carretero et al., showing that tumor-associated eosinophilia contributes to tumor rejection through directed recruitment of tumor-specific CD8 $8^{+} \mathrm{T}$ cells, macrophage polarization, and normalization of the tumor vasculature (44). Whereas the latter were not part of our investigation, $\mathrm{CD}^{+} \mathrm{T}$ cell recruitment to the tumor was not changed in comparison to naive tumors.

Our findings suggest that combined therapy of RT and PD-1 blockade may recruit cytotoxic CD8 ${ }^{+} \mathrm{T}$ cells to the microenvironment of an Lkb1-deficient tumor; however, the expression of the T cell inhibitory markers Lag3 and Tim3 on these $\mathrm{CD}^{+} \mathrm{T}$ cells was significantly increased compared with Kras tumors, potentially indicating reduced cytotoxic activity (Supplemental Figure 4D).

\section{Discussion}

The purpose of this study was to examine the feasibility and efficacy of combined RT and immune checkpoint blockade in a highly predictive animal-based translational cancer research platform utilizing Krasdriven NSCLC-specific GEMMs. Despite a growing body of literature describing in vivo studies of com- 
bined RT and immunotherapy, the majority of these experiments were performed in syngeneic mouse models with ectopic implantation of cancerous cells and potential lack of altered tumor vasculature and concomitantly developed tumor microenvironment $(19,45,46)$. However, the relevance of tissue location in terms of RT-induced immune phenotype changes was previously demonstrated in the ALTS1C1 glioma model (47). RT-mediated clustering of TAM was only seen in intracranial tumors, whereas tumors grown intramuscularly did not show these effects. However, besides our preclinical trial, only one other study employed an autochthonous model (prostate cancer) to examine synergistic effects of RT and immunotherapy, specifically granulocyte/macrophage colony-stimulating factor-secreting cellular immunotherapy (48).

Here, we provide evidence that clinical evaluation of combined RT and immunomodulatory therapy with $\alpha \mathrm{PD}-1$ appears promising for patients with KRAS-driven NSCLC, as our pronounced and durable tumor responses in a highly predictive GEMM are striking. We speculate that the observed tumor responses could potentially be extended beyond $12-17$ weeks in the majority of treated mice, omitting the limitations of our tumor model. As mentioned above, 6 responding mice had to be discontinued owing to contralateral tumor growth, although the dual-treated tumor within the left lung remained small. Although our experimental setting was not designed to analyze the abscopal effect in Kras-driven mNSCLC, we were surprised by this finding, which suggests that using RT on a local tumor nodule to boost systemic immune response together with checkpoint blockade may not work. Given the importance of this observation in regards to clinical translation, careful evaluation of our tumor model is warranted. In contrast to humans, the incidence of metastasis in this tumor model is very low (49). We therefore hypothesize that immediate recovery of the mouse after intrathoracic injection of Adeno-Cre into the left lung lobe causes small amounts of virus to spill into the right lung lobes, which promote tumor growth with an extended latency (8). Furthermore, although these contralateral tumors grow over a longer time period, we believe that these tumors do not harbor mutations other than the Kras ${ }^{G 12 D}$-driver mutation, as these tumor models are considered genetically stable (50). Whether the emergence of these delayed contralateral lesions indicates that the concept of an abscopal effect is either not valid in Kras-driven GEMMs or generally in GEMMs needs much further in-depth evaluation with appropriate experimental design and additional tumor models of other tumor types. Of note, until recently, only case reports, but no large clinical studies, have been published on the systemic immune-related effects of RT (51). Thus, it is possible that this phenomenon is also a rather rare event in humans. As commonly observed in the clinical setting, we also monitored tumor relapse in 2 of 10 mice, despite several weeks of response (Figure 1C and Supplemental Figure 1B). Whether this disease progression could have been influenced by reirradiation should be addressed in future analyses.

The translation of treatment doses and fractionation schemes used in humans to mice is difficult and not very well studied $(8,18)$. Whereas in the majority of in vivo RT studies a single dose is prescribed, Dewan and colleagues specifically analyzed the efficacy of different dose-fractionation regimens with CTLA-4 blockade in two syngeneic mouse models (breast and colorectal cancer) and showed superiority of the multi-fraction versus single-fraction approach (19). We therefore chose to apply one of our previously tested fractionated regimens in these models: tumors were irradiated to a dose of $17 \mathrm{~Gy}(8.5 \mathrm{~Gy} \times 2)(8)$.

The timing for best harnessing the combinatorial effects of RT and immune-stimulating agents is essential, and likely dependent on various factors, such as tumor type, tumor-associated immune microenvironment, and the immunotherapeutic agent itself. In melanoma, aCTLA-4 antibody application prior to RT was shown to enhance antitumor immunity by removal of Tregs (52). However, immunogenic cell death mediated by RT occurs within 1 to 3 days after treatment $(19,53)$. We reasoned that RT-induced immunogenic cell death could be improved by blocking the PD-1/PD-L1 axis shortly after RT administration, as similar therapeutic effects have been observed in a syngeneic colon cancer model using either $\alpha \mathrm{PD}-\mathrm{L} 1$ or $\alpha \mathrm{PD}-1$ antibodies in combination with RT of $10 \mathrm{~Gy}$ in 5 fractions (46). This study further demonstrated increased expression of tumor cell PD-L1 up to 7 days after RT. Additionally, the authors provide evidence that combination of RT with $\alpha$ PD-1 was superior to combination with $\alpha$ PD-L1 (curative in $80 \%$ vs. $66 \%$ ).

The recent review by Vatner and Formenti summarizes the effects of RT on tumor myeloid cells (54). Consistent with their classification, we also observed long-lived recruitment of TAM after RT in some tumors (Figure 3B). However, contrary to prior published preclinical data, we observed major removal of TAM within the first week after RT (Figure 2B) (45). This discrepancy might originate from the different models used in the distinct studies. Furthermore, the definition of TAM likely differs in both studies due to the particular situation in the lung compartment and, therefore, precludes formal comparison. Besides several other subgroups, AMs, which are considered tissue-resident macrophages, represent the predomi- 
nant group and remain viable for prolonged periods (55). Although caution is required when comparing immune cell behavior observed in healthy lung tissue or acute lung injury to phenomena in the cancer context, others have noted that bleomycin-induced injury led to similar marked shrinkage of the AM pool as our RT regimen (56). Importantly, we expected to monitor an increment of neutrophil influx due to the RT-induced inflammation within the tumor microenvironment, which did not occur. An explanation could be that this inflammation-mediated neutrophil recruitment is potentially masked by continuous neutrophil death within 24 to 48 hours after activation in the tumor (57). Alternatively, AMs have been described to prevent neutrophil influx (58). Whether this is a realistic scenario regarding the drastic diminution of TAM remains elusive.

Special emphasis on treatment scheduling, with regard to the efficacy of RT and PD-1/PD-L1 blockade, is warranted in patients with NSCLC to limit adverse reactions. These patients are likely to have poor lung reserve function due to smoking, chronic obstructive pulmonary disease, advanced disease, and prior cancer therapy, and cumulative toxicity of pneumonitis would be of particular concern. RT of lung malignancies almost always inevitably involves normal lung parenchyma, which could potentially lead to variable extents of pulmonary inflammation and radiation pneumonitis (59). Although clinical pneumonitis rates for the $\alpha$ PD-1 antibody nivolumab are similar to or lower than those of other drugs commonly used in NSCLC (docetaxel: 4.6\%, ref. 60; gefitinib: 3.5\%, ref. 61), two patient deaths associated with pneumonitis were reported (62). Whether $\alpha \mathrm{PD}-\mathrm{L} 1$ antibodies have a lower risk of pneumonitis is currently under clinical investigation. Despite the specific predispositions for cumulative toxicity in patients with NSCLC, our preclinical data do not suggest that treatment of RT-refractory $K R A S$-driven tumors with $\alpha \mathrm{PD}$-L1 or $\alpha \mathrm{PD}$ 1 antibodies would be beneficial in the clinic. It remains to be determined how $\alpha \mathrm{PD}-1$ treatment induces increased expression of Lag3, Tim3, and CTLA-4 in RT-relapsed Kras tumors and if concomitant application of neutralizing antibodies against these inhibitory $\mathrm{T}$ cell markers might reverse the therapeutic responsiveness. Given our observations, we hypothesize that acute effects of RT play an essential role in provoking the synergistic treatment outcome. Currently, a few clinical trials assessing the combinatorial effects of RT and immunomodulatory agents in patients with NSCLC have been initiated: RT with ipilimumab (NCT02221739) and RT with granulocyte/macrophage colony-stimulating factor (NCT02623595).

Although we observed durable and prolonged synergistic treatment efficacy of RT and PD-1 blockade in Kras-driven mNSCLC, this effect was limited to $60 \%$ to $80 \%$ of recipient mice and not potent enough to induce tumor regression in Kras/Lkb1 tumors. Our immune analysis indicates that the combinatorial treatment predominantly induces changes within the $\mathrm{T}$ cell compartment resulting in a more antitumor environment. However, increased expression of Lag3 and Tim3 potentially prevent exertion of synergistic therapeutic effects to its fullest extent. Furthermore, the increased accumulation of TAN in Kras/Lkb1 versus Kras tumors (Supplemental Figure 4C) and subsequent secretion of tumor-promoting cytokines might play a critical role in lack of therapeutic efficacy (12). Hence, this finding suggests that addition of further immunomodulatory agents to increase response rates might be valuable, despite the potential risk of side effects. Following the idea of combining RT with multiple checkpoint blockades, Twyman-Saint Victor et al. provided evidence of feasibility and efficacy in patients with metastatic melanoma and verified their observations in mice (63). Briefly, taking advantage of the inhibitory effects of $\alpha$ CTLA- 4 antibodies on Tregs resulting in an augmented $\mathrm{CD}^{+} /$Treg ratio and increased diversity of the $\mathrm{T}$ cell receptor repertoire of intratumoral T cells upon RT, the authors demonstrated that further application of $\alpha \mathrm{PD}-\mathrm{L} 1$ inverted T cell exhaustion and led to improved T cell expansion. This concept of targeting tumor-infiltrating Tregs was previously shown to have pronounced preclinical effects if applied transiently and in combination with RT (64). Furthermore, the relative radioresistance of Tregs in contrast to other lymphocytes argues strongly for the rationale of combined RT and Treg ablation (65).

Although our pharmacodynamic studies of RT and PD-1 blockade in Kras/Lkb1 tumors showed favorable antitumor changes in regards to improved $\mathrm{CD}^{+} /$Treg ratio, this marker alone may not be sufficient to predict therapeutic response. Potential explanations might be that the observed changes compared with the other treatment groups are only modest or that the expression of $\mathrm{T}$ cell inhibitory markers on $\mathrm{CD}^{+} \mathrm{T}$ cells was significantly altered compared with Kras tumors (Supplemental Figure 4D). It remains uncertain what underlying mechanisms exert compensatory signaling such that the milieu remains suppressive of $\mathrm{CD} 8^{+} \mathrm{T}$ cells. Inclusion of additional immunomodulatory drugs could be a logical next step towards enhancing the treatment option for Kras/Lkb1 tumors.

Albeit our current data allow the assumption that transient Treg depletion and/or multiple checkpoint 
blockade against PD-1, Lag3, and Tim3 in addition to RT are pertinent to improve therapeutic outcome of Kras / Lkb1 tumors, one should keep in mind that low-dose RT (single dose $\leq 1.0 \mathrm{~Gy}$ ) may also be utilized to attenuate inflammatory processes (66). Furthermore, Klug et al. demonstrated that low-dose $\gamma$ irradiation led to differentiation of inducible $\mathrm{NOS}^{+} \mathrm{M} 1$ macrophages, subsequent recruitment of cytotoxic $\mathrm{CD} 8^{+} \mathrm{T}$ cells, and tumor cell killing in a spontaneous insulinoma tumor model (67). Whether these phenomena are operative in the case of an inflammatory tumor microenvironment, as observed in Kras/Lkb1 tumors, remains to be explored in further studies.

Additional genomic alterations may possibly also contribute to immune resistance. This is of particular importance in the case of RT-refractory tumors. However, the molecular determinants of immune resistance are currently poorly understood. Two recent studies provide initial evidence that oncogenic pathway signaling may support resistance to antitumor immune response $(68,69)$. Spranger and colleagues found that melanoma cell-intrinsic activation of the $\mathrm{WNT} / \beta$-catenin signaling pathway correlated with lack of intratumoral $\mathrm{T}$ cell infiltration (69). Similar decreased $\mathrm{T}$ cell trafficking into melanomas mediated by expression of immunosuppressive cytokines was observed upon loss of phosphatase and tensin homolog (PTEN) (68). Therapeutic efficacy of $\alpha \mathrm{PD}-1$ and $\alpha \mathrm{CTLA}-4$ antibodies could be improved by additional treatment with a selective PI3K $\beta$ inhibitor in a mouse model. Together, these findings demonstrate that further examination of underlying molecularly driven mechanisms of immune resistance is warranted in the future.

In summary, we provide evidence that clinical evaluation of combined RT and immunomodulatory therapy with $\alpha \mathrm{PD}-1$ appears promising for patients with KRAS-driven NSCLC. We demonstrated that the therapeutic outcome strongly depends on the timing of PD-1 blockade, resulting in continuous tumor growth in RT-refractory Kras tumors upon $\alpha \mathrm{PD}-1$ application. We showed that addition of $\alpha \mathrm{PD}-1$ in these tumors led to enhanced expression of T cell inhibitory markers on tumor-infiltrating T cells. Finally, loss of the tumor suppressor $S t k 11 / L k b 1$ attenuates the synergistic efficacy. Due to the distinct immune phenotype of $\mathrm{Kras} / \mathrm{Lkb1}$ tumors, we suggest alternative immunomodulatory concepts to optimally harness the therapeutic efficacy of RT and the adaptive immune response.

\section{Methods}

Mice. Mouse strains harboring a conditional activating mutation (G12D) at the endogenous Kras locus and conditional $L k b 1$ knockout were described previously (41). Male and female mice of $K r a s^{G 12 D / w t}$ and $\mathrm{Kras}^{G 12 \mathrm{D} / \mathrm{wt}} / L k b 1^{10 x / l o x}$ genotype were used for all experiments. All experimental mice were maintained on a mixed genetic background (C57BL/6, BALB/c, and S129). Single nodule murine lung cancer was induced by intrathoracic injection of Adeno-Cre virus (Gene Transfer Vector Core Facility at the University of Iowa) at the age of 6-8 weeks as described previously (8). Animals were randomly assigned to various treatment groups once tumors had formed as indicated. Irradiation experiments were performed using the small animal radiation research platform at the Dana-Farber Cancer Institute. Irradiation parameters and settings were previously described (8). Radiotherapy was given at a fractionation scheme of 8.5 Gy $\times 2$ on 2 consecutive days. Data regarding unirradiated Kras, RT-treated Kras, and some RT-treated Kras/Lkb1 mice was previously published and are therefore historical (8). However, RT-treated Kras mice used to produce RT-refractory tumors (Figure 3 ) were treated simultaneously as the other experimental arms ( $\alpha \mathrm{PD}-1$ and $\mathrm{RT}+\alpha \mathrm{PD}-1$ in Figure 1) and showed similar tumor growth curves as our previous data (Supplemental Figure 3). Animals that received a single treatment with PD-1 blocking antibodies still underwent the stress of placement on the irradiation platform and also underwent cone beam computed tomography imaging as often as the irradiated mice. Rat anti-PD-1 monoclonal blocking antibodies (clone 29F.1A12, generated in-house) (70) or vehicle (PBS) was given i.p. at a dose of $200 \mu \mathrm{g} / \mathrm{mouse}(8-10 \mathrm{mg} / \mathrm{kg}) 3$ times per week starting 6 hours after the second radiation dose. Tumor growth was evaluated every 2 weeks using MRI imaging until the tumor burden met euthanasia criteria. Tumor volume quantification was performed as described previously (8).

Currently, no standard response criteria exist to evaluate the effect of drug treatment on murine lung tumors. Contrary to human studies, which rely on unidimensional or bidimensional tumor measurements, we used tumor volume measurements to determine tumor treatment response as described previously (71). Briefly, we applied the following criteria: (a) complete response $(\mathrm{CR})=$ target lesion disappeared completely; (b) partial response $(\mathrm{PR})=$ at least $30 \%$ volume reduction of target lesion (compared with the baseline tumor volume); (c) progressive disease $(\mathrm{PD})=$ at least $20 \%$ volume increase of target lesion (compared 
with the baseline tumor volume); and (d) stable disease (SD) = neither sufficient reduction or increase of tumor volume to qualify for $\mathrm{PR}$ or $\mathrm{PD}$, respectively (compared with the baseline tumor volume). These are in keeping with RECIST response criteria used in human clinical studies.

Immune cell isolation and analysis. Tumor and tumor-associated immune cells were isolated, identified, and counted as previously described (9), with some modifications. Briefly, after blood and bronchoalveolar lavage fluid collection, lungs were perfused with cold PBS containing $5 \mathrm{mmol} / 1$ EDTA from the right ventricle. Tumors from the left lung lobe were resected and used for histologic, flow cytometry, and immune cell gene expression analysis, respectively. The majority of the tumor sample was used for flow cytometry analysis. Total cell count was divided by tumor weight utilized for each assay. AccuCheck Counting Beads (Invitrogen) were used according to the manufacturer's protocol to count absolute numbers of immune cell populations. After dissociation of the tumor tissue, the cells were treated with rbc lysis (BioLegend) and used directly for staining. Prior to surface marker staining, isolated cells were stained using the LIVE/ DEAD fixable dead cell stain kit (Invitrogen). A complete list of antibodies for flow cytometry analysis can be found in Supplemental Table 1. Detection of anti-PD-1 antibody binding was performed using anti-rat IgG2a (r2a-21B2: eBioscience) without prior Fc blocking (BioLegend), which was performed for all other stainings. All antibodies were used at 1:50 dilution. Intracellular staining for CTLA-4 and Foxp3 was performed according to the manufacturer's protocol (eBioscience). Cell analysis was examined with the use of a BD FACS Canto II cytometer equipped with Diva software (version 8.0) and FlowJo (version X, Tree Star). The gating strategy for immune cell population analysis was previously described (12). Briefly, after gating live, single $\mathrm{CD} 45^{+}$cells from total tumors, cells were differentiated in 3 distinct populations: (a) the lymphocyte population $\left(\mathrm{CD} 11 \mathrm{c}^{-} \mathrm{CD} 11 \mathrm{~b}^{-}\right)$, which is mainly composed of $\mathrm{CD} 4^{+} \mathrm{T}$ cells, $\mathrm{CD} 8^{+} \mathrm{T}$ cells, B cells, and NK cells; (b) a population of $\mathrm{CD} 11 \mathrm{c}^{+} \mathrm{CD} 11 \mathrm{~b}^{-}$cells, which include $\mathrm{CD}^{-} \mathrm{3}^{-}$alveolar macrophages (AMs) and $\mathrm{CD} 103^{+} \mathrm{CD} 11 \mathrm{c}^{+} \mathrm{DCs}$; and (c) a population of $\mathrm{CD} 11 \mathrm{~b}^{+}$cells, which includes $\mathrm{Ly}-6 \mathrm{G}^{+}$neutrophils $\left(\mathrm{CXCR}^{+}\right)$and a $\mathrm{Ly}-6 \mathrm{G}^{-}$population. The $\mathrm{CD} 11 \mathrm{~b}^{+} \mathrm{Ly}-6 \mathrm{G}^{-}$population contains subpopulations, such as SiglecF ${ }^{+} \mathrm{CCR}^{+}$eosinophils and SiglecF-Ly-6 $\mathrm{C}^{\text {hi }}$ monocytes (Supplemental Figure 5).

NanoString experiments. Total cellular RNA was extracted from homogenized tumor tissues using TRIzol reagent (Invitrogen). It was then DNase treated using an Ambion Kit (Ambron) and column purified using a RNeasy Mini kit (QIAGEN). Gene expression profiling was performed on 50-75 ng of RNA using the NanoString Immune Profiling Kit (NanoString Technologies). Capture and Reporter Code sets were added to the samples following the manufacturer's protocol and allowed to hybridize at $65^{\circ} \mathrm{C}$ for 16 hours. Samples were washed and loaded onto a cartridge using the nCounter Analysis System Prep Station per the manufacturer's protocol. The cartridge was scanned using the nCounter Digitical Analyzer at the maximum resolution of 1,150 fields of view. Data Analysis was completed using nSolver software (NanoString Technologies).

Statistics. Statistical analysis was performed with GraphPad Prism (version 6.0d). Differences between the groups were evaluated by 2-tailed unpaired Student's $t$ test, 1-way ANOVA with Tukey's multiple comparisons test, and log-rank test corrected for multiple comparisons with Bonferroni threshold where appropriate. Correlation was evaluated using Pearson's correlation coefficient. Data are presented as mean \pm SEM, and $P$ values of less than 0.05 were considered statistically significant.

Study approval. All in vivo experiments performed in this study were approved by the Animal Care and Use Committee of the Dana-Farber Cancer Institute and the Animal Care and Use Committee of Beth Israel Deaconess Medical Center.

\section{Author contributions}

GSHS, SK, PSH, ACK, and KKW conceived and designed the experiments. GSHS, SK, HK, JH, KAB, JD, AKG, SU, KR, JDC, NPN, and CLC performed the experiments. GSHS, SK, YYL, CLC, and JMH collected and analyzed data. GSHS, SK, PSH, ACK, and KKW wrote the manuscript. GMM, FSH, GJF, and GD gave technical support and provided reagents and conceptual advice. All authors contributed to the final manuscript.

\section{Acknowledgments}

We thank P. Gao for technical assistance, C. Almonte and K. Labbe for administrative assistance, and the members of the Wong, Hammerman, and Kimmelman laboratories for helpful discussions. We thank P. Schiller for help with the biostatistical analysis and A. Scheel for help with the NanoString analysis. This work was supported by the Deutsche Forschungsgemeinschaft (HE 6897/1-1 to G.S. Herter-Sprie and 
HE 6810/1-1 to J.M. Herter), the Else Kroner-Fresenius-Stiftung (2014_A80 to J.M. Herter), the Damon Runyon Cancer Research Foundation (to P.S. Hammerman), the Starr Consortium for Cancer Research (to P.S. Hammerman), the National Cancer Institute (R01 CA195740, CA163896, CA166480, CA122794, and CA140594 to K.K. Wong; CA205150 to P.S. Hammerman; R01CA15749 and R01CA188048 to A.C. Kimmelman), the National Institutes of Health (GM095567 to A.C. Kimmelman), the Stand Up to Cancer Lung Cancer Dream Team Award (to P.S. Hammerman and K.K. Wong), the American Cancer Society research scholar grant (RSG-13-298-01-TBG to A.C. Kimmelman), the Lustgarten Foundation (to A.C. Kimmelman), the Thoracic Foundation (K.K. Wong), the Gross-Loh Family Fund for Lung Cancer Research, and the Susan Spooner Family Lung Cancer Research Fund at Dana-Farber Cancer Institute (K.K. Wong).

Address correspondence to: Alec C. Kimmelman, NYU Langone Medical Center, 560 First Avenue, 2nd FL, Radiation Oncology Department, New York, New York, 10016, USA. Phone: 646.501.8940; E-mail: alec.kimmelman@nyumc.org. Or to: Kwok-Kin Wong, Department of Medical Oncology, Dana-Farber Cancer Institute, 450 Brookline Avenue, Boston, Massachusetts, 02215-5450, USA. Phone: 617.632.6084; E-mail: kwong1@partners.org.

Shohei Koyama's present address is: Division of Respiratory Medicine, Department of Respiratory Medicine, Allergy, and Rheumatic Disease, Osaka University Graduate School of Medicine, Osaka, Japan.

Glenn Dranoff's present address is: Novartis Institutes for BioMedical Research, Cambridge, Massachusetts, USA.

Alec C. Kimmelman's present address is: Department of Radiation Oncology, Perlmutter Cancer Center, New York University, School of Medicine, New York, New York, USA.

1. Siegel R, Ma J, Zou Z, Jemal A. Cancer statistics, 2014. CA Cancer J Clin. 2014;64(1):9-29.

2. Aupérin A, et al. Meta-analysis of concomitant versus sequential radiochemotherapy in locally advanced non-small-cell lung cancer. J Clin Oncol. 2010;28(13):2181-2190.

3. Barcellos-Hoff MH, Park C, Wright EG. Radiation and the microenvironment - tumorigenesis and therapy. Nat Rev Cancer. 2005;5(11):867-875.

4. Topalian SL, et al. Safety, activity, and immune correlates of anti-PD-1 antibody in cancer. NEngl J Med. 2012;366(26):24432454.

5. Brahmer JR, et al. Phase I study of single-agent anti-programmed death-1 (MDX-1106) in refractory solid tumors: safety, clinical activity, pharmacodynamics, and immunologic correlates. J Clin Oncol. 2010;28(19):3167-3175.

6. Rizvi NA, et al. Cancer immunology. Mutational landscape determines sensitivity to PD-1 blockade in non-small cell lung cancer. Science. 2015;348(6230):124-128.

7. Chen Z, et al. A murine lung cancer co-clinical trial identifies genetic modifiers of therapeutic response. Nature. 2012;483(7391):613-617.

8. Herter-Sprie GS, et al. Image-guided radiotherapy platform using single nodule conditional lung cancer mouse models. Nat Commun. 2014;5:5870.

9. Akbay EA, et al. Activation of the PD-1 pathway contributes to immune escape in EGFR-driven lung tumors. Cancer Discov. 2013;3(12):1355-1363.

10. Skoulidis F, et al. Co-occurring genomic alterations define major subsets of KRAS-mutant lung adenocarcinoma with distinct biology, immune profiles, and therapeutic vulnerabilities. Cancer Discov. 2015;5(8):860-877.

11. Schabath MB, et al. Differential association of STK11 TP53 with KRAS mutation-associated gene expression, proliferation immune surveillance in lung adenocarcinoma [published online ahead of print October 19, 2015]. Oncogene. doi: 10.1038/ onc.2015.375.

12. Koyama S, et al. STK11/LKB1 deficiency promotes neutrophil recruitment and proinflammatory cytokine production to suppress T-cell activity in the lung tumor microenvironment. Cancer Res. 2016;76(5):999-1008.

13. Formenti SC. Is classical stereotactic radiotherapy the optimal partner for immunotherapy?. Oncology (Williston Park, NY). $2015 ; 29(5): 340,347,387$.

14. Siva S, et al. Abscopal [corrected] effects after conventional and stereotactic lung irradiation of non-small-cell lung cancer. $J$ Thorac Oncol. 2013;8(8):e71-e72.

15. Golden EB, Demaria S, Schiff PB, Chachoua A, Formenti SC. An abscopal response to radiation and ipilimumab in a patient with metastatic non-small cell lung cancer. Cancer Immunol Res. 2013;1(6):365-372.

16. Postow MA, et al. Immunologic correlates of the abscopal effect in a patient with melanoma. N Engl J Med. 2012;366(10):925931.

17. Stamell EF, Wolchok JD, Gnjatic S, Lee NY, Brownell I. The abscopal effect associated with a systemic anti-melanoma immune response. Int J Radiat Oncol Biol Phys. 2013;85(2):293-295.

18. Perez BA, et al. Assessing the radiation response of lung cancer with different gene mutations using genetically engineered mice. 
Front Oncol. 2013;3:72.

19. Dewan MZ, et al. Fractionated but not single-dose radiotherapy induces an immune-mediated abscopal effect when combined with anti-CTLA-4 antibody. Clin Cancer Res. 2009;15(17):5379-5388.

20. Harris TJ, et al. Radiotherapy augments the immune response to prostate cancer in a time-dependent manner. Prostate. 2008;68(12):1319-1329.

21. Wolchok JD, et al. Guidelines for the evaluation of immune therapy activity in solid tumors: immune-related response criteria Clin Cancer Res. 2009;15(23):7412-7420.

22. Nishino M, et al. Personalized tumor response assessment in the era of molecular medicine: cancer-specific and therapy-specific response criteria to complement pitfalls of RECIST. AJR Am J Roentgenol. 2012;198(4):737-745.

23. Nishino M, Giobbie-Hurder A, Gargano M, Suda M, Ramaiya NH, Hodi FS. Developing a common language for tumor response to immunotherapy: immune-related response criteria using unidimensional measurements. Clin Cancer Res. 2013;19(14):3936-3943.

24. Hodi FS, et al. Improved survival with ipilimumab in patients with metastatic melanoma. N Engl J Med. 2010;363(8):711-723.

25. Hodi FS, Dranoff G. The biologic importance of tumor-infiltrating lymphocytes. J Cutan Pathol. 2010;37 Suppl 1:48-53.

26. Mahmoud SM, et al. Tumor-infiltrating CD8+ lymphocytes predict clinical outcome in breast cancer. J Clin Oncol. 2011;29(15):1949-1955.

27. Galon J, et al. Type, density, and location of immune cells within human colorectal tumors predict clinical outcome. Science 2006;313(5795):1960-1964.

28. Zhang L, et al. Intratumoral T cells, recurrence, and survival in epithelial ovarian cancer. NEngl J Med. 2003;348(3):203-213.

29. Curiel TJ, et al. Specific recruitment of regulatory T cells in ovarian carcinoma fosters immune privilege and predicts reduced survival. Nat Med. 2004;10(9):942-949.

30. Gao Q, et al. Intratumoral balance of regulatory and cytotoxic T cells is associated with prognosis of hepatocellular carcinoma after resection. J Clin Oncol. 2007;25(18):2586-2593.

31. Bauer M, Goldstein M, Christmann M, Becker H, Heylmann D, Kaina B. Human monocytes are severely impaired in base and DNA double-strand break repair that renders them vulnerable to oxidative stress. Proc Natl Acad Sci USA. 2011;108(52):2110521110.

32. Conrad S, Ritter S, Fournier C, Nixdorff K. Differential effects of irradiation with carbon ions and x-rays on macrophage function. J Radiat Res. 2009;50(3):223-231.

33. Toivonen $\mathrm{P}$, Kivelä T. Infiltrating macrophages in extratumoural tissues after brachytherapy of uveal melanoma. Acta Ophthalmol. 2012;90(4):341-349.

34. Julow J, Szeifert GT, Bálint K, Nyáry I, Nemes Z. The role of microglia/macrophage system in the tissue response to I-125 interstitial brachytherapy of cerebral gliomas. Neurol Res. 2007;29(3):233-238.

35. Crittenden MR, Cottam B, Savage T, Nguyen C, Newell P, Gough MJ. Expression of NF- $\mathrm{kB}$ p50 in tumor stroma limits the control of tumors by radiation therapy. PLoS ONE. 2012;7(6):e39295.

36. Lugade AA, Moran JP, Gerber SA, Rose RC, Frelinger JG, Lord EM. Local radiation therapy of B16 melanoma tumors increases the generation of tumor antigen-specific effector cells that traffic to the tumor. J Immunol. 2005;174(12):7516-7523.

37. Kioi M, Vogel H, Schultz G, Hoffman RM, Harsh GR, Brown JM. Inhibition of vasculogenesis, but not angiogenesis, prevents the recurrence of glioblastoma after irradiation in mice. J Clin Invest. 2010;120(3):694-705.

38. Cavnar MJ, et al. KIT oncogene inhibition drives intratumoral macrophage M2 polarization. J Exp Med. 2013;210(13):28732886.

39. Ding L, et al. Somatic mutations affect key pathways in lung adenocarcinoma. Nature. 2008;455(7216):1069-1075.

40. Gill RK, et al. Frequent homozygous deletion of the LKB1/STK11 gene in non-small cell lung cancer. Oncogene. 2011;30(35):3784-3791.

41. Ji H, et al. LKB1 modulates lung cancer differentiation and metastasis. Nature. 2007;448(7155):807-810.

42. Calles A, et al. Immunohistochemical loss of LKB1 is a biomarker for more aggressive biology in KRAS-mutant lung adenocarcinoma. Clin Cancer Res. 2015;21(12):2851-2860.

43. Zhao N, et al. Alterations of LKB1 and KRAS and risk of brain metastasis: comprehensive characterization by mutation analysis, copy number, and gene expression in non-small-cell lung carcinoma. Lung Cancer. 2014;86(2):255-261.

44. Carretero R, et al. Eosinophils orchestrate cancer rejection by normalizing tumor vessels and enhancing infiltration of CD8(+) T cells. Nat Immunol. 2015;16(6):609-617.

45. Deng L, et al. Irradiation and anti-PD-L1 treatment synergistically promote antitumor immunity in mice. J Clin Invest. 2014;124(2):687-695

46. Dovedi SJ, et al. Acquired resistance to fractionated radiotherapy can be overcome by concurrent PD-L1 blockade. Cancer Res. 2014;74(19):5458-5468.

47. Chiang CS, et al. Irradiation promotes an $\mathrm{m} 2$ macrophage phenotype in tumor hypoxia. Front Oncol. 2012;2:89.

48. Wada S, et al. Combined treatment effects of radiation and immunotherapy: studies in an autochthonous prostate cancer model. Int J Radiat Oncol Biol Phys. 2013;87(4):769-776.

49. Jackson EL, et al. Analysis of lung tumor initiation and progression using conditional expression of oncogenic K-ras. Genes Dev. 2001;15(24):3243-3248.

50. Heyer J, Kwong LN, Lowe SW, Chin L. Non-germline genetically engineered mouse models for translational cancer research. Nat Rev Cancer. 2010;10(7):470-480.

51. Reynders K, Illidge T, Siva S, Chang JY, De Ruysscher D. The abscopal effect of local radiotherapy: using immunotherapy to make a rare event clinically relevant. Cancer Treat Rev. 2015;41(6):503-510.

52. Simpson TR, et al. Fc-dependent depletion of tumor-infiltrating regulatory T cells co-defines the efficacy of anti-CTLA-4 therapy against melanoma. J Exp Med. 2013;210(9):1695-1710.

53. Golden EB, Frances D, Pellicciotta I, Demaria S, Helen Barcellos-Hoff M, Formenti SC. Radiation fosters dose-dependent and chemotherapy-induced immunogenic cell death. Oncoimmunology. 2014;3:e28518.

54. Vatner RE, Formenti SC. Myeloid-derived cells in tumors: effects of radiation. Semin Radiat Oncol. 2015;25(1):18-27. 
55. Yona S, et al. Fate mapping reveals origins and dynamics of monocytes and tissue macrophages under homeostasis. Immunity. 2013;38(1):79-91

56. Misharin AV, Morales-Nebreda L, Mutlu GM, Budinger GR, Perlman H. Flow cytometric analysis of macrophages and dendritic cell subsets in the mouse lung. Am J Respir Cell Mol Biol. 2013;49(4):503-510.

57. O'Donnell JA, et al. Fas regulates neutrophil lifespan during viral and bacterial infection. J Leukoc Biol. 2015;97(2):321-326.

58. Beck-Schimmer B, Schwendener R, Pasch T, Reyes L, Booy C, Schimmer RC. Alveolar macrophages regulate neutrophil recruitment in endotoxin-induced lung injury. Respir Res. 2005;6:61.

59. Palma DA, Senan S, Haasbeek CJ, Verbakel WF, Vincent A, Lagerwaard F. Radiological and clinical pneumonitis after stereotactic lung radiotherapy: a matched analysis of three-dimensional conformal and volumetric-modulated arc therapy techniques. Int J Radiat Oncol Biol Phys. 2011;80(2):506-513.

60. Grande C, Villanueva MJ, Huidobro G, Casal J. Docetaxel-induced interstitial pneumonitis following non-small-cell lung cancer treatment. Clin Transl Oncol. 2007;9(9):578-581.

61. Konishi J, et al. Analysis of the response and toxicity to gefitinib of non-small cell lung cancer. Anticancer Res. 2005;25(1B):435441.

62. Sundar R, Cho BC, Brahmer JR, Soo RA. Nivolumab in NSCLC: latest evidence and clinical potential. Ther Adv Med Oncol. 2015;7(2):85-96.

63. Twyman-Saint Victor C, et al. Radiation and dual checkpoint blockade activate non-redundant immune mechanisms in cancer. Nature. 2015;520(7547):373-377.

64. Bos PD, Plitas G, Rudra D, Lee SY, Rudensky AY. Transient regulatory T cell ablation deters oncogene-driven breast cancer and enhances radiotherapy. J Exp Med. 2013;210(11):2435-2466.

65. Komatsu N, Hori S. Full restoration of peripheral Foxp3 ${ }^{+}$regulatory T cell pool by radioresistant host cells in scurfy bone marrow chimeras. Proc Natl Acad Sci USA. 2007;104(21):8959-8964

66. Frey B, Hehlgans S, Rödel F, Gaipl US. Modulation of inflammation by low and high doses of ionizing radiation: Implications for benign and malign diseases. Cancer Lett. 2015;368(2):230-237.

67. Klug F, et al. Low-dose irradiation programs macrophage differentiation to an iNOS ${ }^{+} / \mathrm{M} 1$ phenotype that orchestrates effective T cell immunotherapy. Cancer Cell. 2013;24(5):589-602.

68. Peng W, et al. Loss of PTEN promotes resistance to T cell-mediated immunotherapy. Cancer Discov. 2016;6(2):202-216.

69. Spranger S, Bao R, Gajewski TF. Melanoma-intrinsic $\beta$-catenin signalling prevents anti-tumour immunity. Nature. 2015;523(7559):231-235.

70. Barber DL, et al. Restoring function in exhausted CD8 T cells during chronic viral infection. Nature. 2006;439(7077):682-687.

71. Regales L, et al. Development of new mouse lung tumor models expressing EGFR T790M mutants associated with clinical resistance to kinase inhibitors. PLoS ONE. 2007;2(8):e810. 